\title{
Structural basis of malaria transmission blockade by a monoclonal antibody to gamete fusogen HAP2
}

Juan Feng ${ }^{1,2}$, Xianchi Dong ${ }^{1,2}$, Adam DeCosta ${ }^{1}$, Yang $\mathrm{Su}^{1,2}$, Fiona Angrisano ${ }^{3,4}$, Katarzyna A. Sala ${ }^{3}$, Andrew M. Blagborough $^{3}$, Chafen $\mathrm{Lu}^{1,2 *}$ and Timothy A. Springer ${ }^{1,2 *}$

\section{Affiliations}

${ }^{1}$ Program in Cellular and Molecular Medicine, Children's Hospital Boston, Boston, MA

${ }^{2}$ Department of Biological Chemistry and Molecular Pharmacology and Department of Pediatrics, Boston Children's Hospital and Harvard Medical School, Boston, MA

${ }^{3}$ Department of Pathology, University of Cambridge, Tennis Court Road, Cambridge, UK, CB1 1QP

${ }^{4}$ The Macfarlane Burnet Institute for Medical and Public Health, Melbourne, Australia, 3004

*Chafen Lu: 1u@crystal.harvard.edu

*Timothy Springer: springer@,crystal.harvard.edu

Key Words: HAP2/malaria transmission-blocking vaccine/membrane fusion/gamete fusogen

Abstract. HAP2 is a transmembrane gamete fusogen found in multiple eukaryotic kingdoms and is structurally homologous to viral class II fusogens. Studies in Plasmodium have suggested that HAP2 is an attractive target for vaccines that block transmission of malaria. HAP2 has three extracellular domains, arranged in the order D2, D1, and D3. Here, we report monoclonal antibodies against the D3 fragment of Plasmodium berghei HAP2 and crystal structures of D3 in complex with Fab fragments of two of these antibodies, one of which blocks fertilization of Plasmodium berghei in vitro and transmission of malaria in mosquitos. We also show how this Fab binds the complete HAP2 ectodomain with electron microscopy. The two antibodies crossreact with HAP2 among multiple plasmodial species. Our characterization of the Plasmodium D3 structure, HAP2 ectodomain architecture, and mechanism of inhibition provide insights for the development of a vaccine to block malaria transmission. 


\section{INTRODUCTION}

HAP2, a gamete fusogen required for fertilization, is found in eukaryotic plant, metazoan, and protozoan kingdoms (1). Crystal structures are known of HAP2 from the unicellular green alga, Chlamydomonas reinhardti; a model plant, Arabidopsis thaliana; and a protozoan parasite, Trypanosoma cruzi (2-5). HAP2 can form a trimeric structure that is structurally homologous to the fusion state of viral class II fusion proteins. These fusogens have three extracellular domains, arranged in the order D2, D1, and D3, a disordered stem region, a single-pass transmembrane domain, and a cytoplasmic domain, and are expressed on the plasma membrane of male gametes or the envelope of viruses (Fig. 1).

HAP2 is required for fertilization in Plasmodium parasites $(6,7)$ and is thus an attractive target for vaccines that block transmission of malaria. Gamete fertilization takes place in the mosquito midgut in a blood meal taken from an infected individual. Blocking fertilization prevents transmission to the individual from which the mosquito takes its second, final blood meal. Plasmodium gametes emerge from infected erythrocytes well before digestion of the blood meal; specific antibodies present in the blood meal will react with and can agglutinate or otherwise neutralize gametes (8-11). HAP2 is present on male gametes (microgametes) and has hydrophobic fusion loops at the tip of D2 that insert into the plasma membrane of the female macrogamete and trigger trimerization and structural rearrangements of HAP2 to the fusion state, which mediates membrane fusion and merging of the cytoplasm of the two gametes through a fusion pore (12) (Fig. 1). To mediate fusion, D3 of HAP2 folds over an inner trimeric core composed of D1 and D2 (Fig. 1). We reasoned that antibodies to D3 of HAP2 that blocked the interface formed with D1 and D2 in the fusion state might block fusion, as has been demonstrated with viral fusogens (13-15). Thus, we hypothesized that HAP2 D3 antibody taken up in a blood meal could inhibit fertilization and infection of the mosquito and subsequent transmission to humans (16) (Fig. 1).

Immunization of mice with HAP2 fragments or putative HAP2 fusion loop peptides have proved the concept that antibodies to different domains of HAP2 can, to different extents, block transmission of Plasmodium berghei or P. vivax, species that infect rodents and humans, respectively (8-10). However, we are far from having an optimized HAP2 immunogen. Furthermore, the high sequence divergence of HAP2 in Plasmodium species from structurally characterized HAP2 in other phyla emphasizes the importance of Plasmodium HAP2 structures for rational vaccine design. Here, to advance a vaccine that would block malaria transmission, we have expressed a D3 fragment of $P$. berghei HAP2, raised monoclonal antibodies, crystallized D3 in complex with Fab fragments of two antibodies, and examined Fab complexes with the complete HAP2 ectodomain (D1-D3) by negative stain electron microscopy (EM). Furthermore, we show that one of these antibodies potently blocks gamete fertilization and transmission and thus for the first time that a monoclonal antibody to HAP 2 can block transmission. We define the advantages, as well as the limitations, of using the D3 domain of HAP2 as an immunogen for transmission blockade. Moreover, the two structurally characterized antibodies have cross-reactivity with HAP2s among multiple plasmodial species that can cause malaria in humans. The insights into Plasmodium D3 structure, HAP2 ectodomain architecture, and mechanism of inhibition are important steps toward the development of a vaccine to block malaria transmission.

\section{RESULTS}

\section{P. berghei HAP2 D3 elicits antibodies that cross react with human malaria pathogens}

Apicomplexans such as Plasmodium are eukaryotes that have extracellular proteins that are disulfidelinked and glycosylated; therefore, we produced HAP2 proteins in insect and mammalian cells which are competent for such modifications. C-mannosylation and O-glycosylation in Plasmodium have been verified; however, while Plasmodium species have been suggested to have unusually short N-glycans, N-glycosylation has yet to be verified with any specific protein (17-21). To elicit antibodies, we expressed in Drosophila S2 cells a $P$. berghei HAP2 D3 construct (residues 477-621, containing 4 putative N-glycosylation sites) containing a Cterminal His tag. After Ni-affinity chromatography, the material was nearly homogenous, as shown by SDSPAGE and Coomassie blue staining (Fig. 2A, lane 1). Shaving the N-glycans with endoglycosidase D (Endo D) followed by gel filtration decreased size and heterogeneity in SDS-PAGE and confirmed N-glycosylation of D3 (Fig 2A, lane 2).

To produce monoclonal antibodies (mAbs), mice were immunized with the glycan-shaved D3. Antibodies elicited to the shaved protein, which contains one N-glycan residue at each N-glycosylation site, would be expected to react with D3 without being influenced by its glycosylation status. Five $\operatorname{IgG} \mathrm{mAbs}$ reacted with the 
immunogen with nanomolar EC50 values in ELISA (Fig 2B). Surface plasmon resonance (SPR) showed that Fabs of two mAbs, 2/6.14 and 2/1.12, bind with affinities of 5 to $10 \mathrm{nM}$ (Supplementary Fig. 1). The antibodies also immunoprecipitated a shorter D3 (502-617) with all putative N-glycosylation sites mutated out (Fig 2C, top panel). Immunoprecipitation was comparable to the His-tag antibody, showing that the epitopes of the $5 \mathrm{mAbs}$ resided within residues 502-617 and were unaffected by N-glycosylation. One antibody (1/5.13) that reacted with the C-terminal His tag and is comparable in specificity and sensitivity to commercial His tag antibodies is described in Methods.

HAP2 D3 was also expressed and shaved with Endo D from Plasmodium species that can infect humans: P. knowlesi, P. vivax, P. malariae, P. ovale and P. falciparum (Fig. 2A). As their D3 domains share 60-70\% amino acid sequence identity with $P$. berghei, we tested cross-reactivity with the mAbs to $P$. berghei $\mathrm{D} 3$ using immunoprecipitation (Fig 2C). We quantified immunoprecipitaton (Fig 2D) and also measured cross-reactivity using ELISA (Fig. 2E and Supplemental Fig. S3). mAbs 2/6.14 and 2/1.12 were especially efficient at recognizing D3 from multiple species (Fig 2C). mAb 2/6.14 pulled down about $70 \%, 30 \%$ and $20 \%$ of $P$. knowlesi, $P$. ovale, and $P$. malariae D3, respectively, and $\mathrm{mAb} 2 / 1.12$ was even more reactive, pulling down $60-100 \%$ of $\mathrm{D} 3$ from all species except $P$. falciparum D3 (Fig 2C and Fig 2D). In contrast, mAb 2/1.40 showed strong reactivity to $P$. ovale, but not to D3 of other species, while mAbs $2 / 3.3$ and 2/4.36 were highly specific for $P$. berghei (Fig 2C and Fig 2D). mAbs 2/6.14 and 2/1.12 bound all species of D3 tested, as shown by ELISA EC50 values in the 1 to $1000 \mathrm{nM}$ range (Fig. 2E and Supplementary Fig. S2). Strong binding of $P$. knowlesi D3 to both 2/6.14 and 2/1.12 was observed with EC50 of $<10 \mathrm{nM}$.

\section{Antibody reactivity with gametes and blockade of fertilization in vitro and in vivo}

Two individual assays with gametes, immunofluorescent staining with mAbs and ookinete conversion, were carried out in vitro. The change of environment from the bloodstream to the mosquito midgut triggers the development of gametocytes in infected erythrocytes into mature, highly motile "male" microgametes and more sessile "female" macrogametes and gamete emergence from erythrocytes. These events can be mimicked in vitro by reducing the temperature or $\mathrm{pH}$ and adding xanthurenic acid to the medium (ookinete medium). Each male gametocyte gives rise to eight microgametes, which look like a number of waving cilia as they emerge from the infected erythrocyte; this process is thus termed "exflagellation".

Reactivity of mAbs to native HAP2 in P. berghei microgametes was determined using cultured, infected erythrocytes undergoing exflagellation. After cultures were fixed, gametes adsorbed to slides were incubated with mouse $\mathrm{D} 3 \mathrm{mAb}$ and rabbit anti-alpha-tubulin followed by staining with fluorochrome-conjugated secondary antibodies specific for mouse and rabbit IgG and DAPI. Anti-tubulin and DAPI were used to identify microgametes by their highly elongated microtubule cytoskeletons and nuclei. All 5 D3 mAbs specifically stained P. berghei microgametes (Fig 3A).

We next tested the ability of the D3 mAbs to inhibit $P$. berghei microgametes from fertilizing macrogametes and forming ookinetes in vitro. Mouse blood infected with $P$. berghei gametocytes was mixed with D3 mAbs or control mouse IgG diluted in ookinete medium. After $24 \mathrm{~h}$, ookinetes and unfertilized female gametocytes were identified by their morphology and staining with a fluorescent antibody, counted, and ookinete conversion rates were calculated. Only a single microgamete is required to fertilize a macrogamete, and each ookinete observed comprises a successful fertilization event. In control IgG, conversion rates were $\sim 80 \%$ (Fig. 3B). Strikingly, mAb $2 / 6.14$ at $500 \mu \mathrm{g} / \mathrm{ml}$ completely inhibited ookinete conversion and at $250 \mu \mathrm{g} / \mathrm{ml}$ inhibited by $96 \%(\mathrm{p}<0.001)$. In contrast, none of the other $\mathrm{mAbs}$ inhibited by $>50 \%$, although $\mathrm{mAb} 2 / 1.40$ showed significant inhibition at $500 \mu \mathrm{g} / \mathrm{ml}$ but not at $250 \mu \mathrm{g} / \mathrm{ml}$ (Fig 3B).

We then tested mAb 2/6.14 for its ability to block transmission in vivo in mosquitoes. Female Anopheles stephensi mosquitoes were allowed to feed through membranes on blood from $P$. berghei infected mice mixed with $\mathrm{mAb} 2 / 6.14$ or control IgG. After 14 days, mosquito midguts were dissected and oocysts/midgut were counted, i.e., oocyst intensity was determined. Oocyst intensity was significantly reduced by mAb $2 / 6.14$, particularly at 250 and $100 \mu \mathrm{g} / \mathrm{ml}$ (Fig. 3C). We also measured the prevalence of infection among the fed mosquitoes, i.e. the $\%$ of mosquitoes with at least one oocyst. Prevalence was significantly reduced by $\mathrm{mAb}$ $2 / 6.14$ at both 250 and $100 \mu \mathrm{g} / \mathrm{ml}$ (Supplementary Fig. S3). Summing the results over all mosquitos in the three independent experiments showed significant inhibition of intensity at all three $\mathrm{mAb} 2 / 6.14$ concentrations, significant inhibition of prevalence at 250 and $100 \mu \mathrm{g} / \mathrm{ml}$ of $\mathrm{mAb} 2 / 6.14$, dose-dependent reduction of both 
measures over the three antibody concentrations, and a reduction of intensity by $85 \%$ at $250 \mu \mathrm{g} / \mathrm{ml}$ (Fig. 3D). Overall, the results show that all five antibodies react with microgametes, that $\mathrm{mAb} 2 / 6.14$ exhibits potent ability to block $P$. berghei fertilization in vitro and in vivo, and thus that purified HAP2 D3 has potential as a transmission-blocking vaccine.

\section{Structural characterization of $P$. berghei HAP2 and complexes of D3 with Fabs}

To obtain structural insights relevant to rational development of a transmission-blocking vaccine, we obtained crystal structures of the P. berghei HAP2 D3 502-617 fragment with its three N-linked sites mutated out in complex with Fab fragments. Diffraction data was collected and refined to resolutions of 2.8 and $2.1 \AA$ with Rfree of 29\% and 23\% for complexes with the transmission-blocking Fab 2/6.14 and cross-reactive Fab 2/1.12, respectively (Table S1). Each structure has two independent D3-Fab complexes in the asymmetric unit, giving us four examples of D3. The two Fab fragments bind to opposite faces of D3 (Fig. 4A).

D3 contains seven $\beta$-strands labeled $A$ to $E$ that are arranged into two $\beta$-sheets containing the $\mathrm{ABE}$ and DCFG $\beta$-strands (Fig. 4A and B). These $\beta$-sheets associate through hydrophobic faces to form a $\beta$-sandwich. The way in which the sequence folds into this arrangement classifies it as a fibronectin type III (Fn3) domain. The D3 $\beta$-strands connect to one another through loops at each end of the domain. The A-B, C-D, and F-G loops link adjacent $\beta$-strands within a sheet and the B-C, D-E, and E-F loops link the two sheets.

Comparisons among the four examples of D3 show markedly different conformations for the loops at the C-terminal end of D3, which abut the polypeptide segment that connects to the plasma membrane (Fig. 4B). Quantitation of flexibility in these loops, A-B, C-D and E-F, shows high RMSD values or lack of comparison because of residues missing in density (dashes, Fig. 4E). In contrast, the loops at the N-terminal end that abut D1 show very similar conformations in all independent crystallographic examples. These loops, B-C, D-E, and F-G, show low RMSD values (Fig. 4E), consistent with the presence of several backbone-backbone or sidechainbackbone hydrogen bonds that stabilize each loop. Three sidechains that support the hydrogen bond networks in these loops, Asn-531 and Asn-532 in the B-C loop and Asp-598 in the FG-loop (overlined in Fig. 4E), are invariant in the 6 Plasmodia species.

The two antibodies bind largely to opposite faces of D3, with 2/1.12 binding primarily to the ABE sheet and 2/6.14 binding primarily to the DCFG sheet (Fab contacts are colored in green and red, respectively in Fig. 4A). The overall shapes of the contact surfaces are shown in open book views in which the Fab and D3 are rotated apart like two facing pages in an open book (Fig. 4C and D). In each Fab, both the heavy and light chain variable domains contribute significantly to the contact with D3. The sidechains with major contacts are shown as large colored circles over the alignment in Fig. 4E and shown in stick in Fig. 4F and G.

Although the 2/1.12 Fab primarily engages the ABE $\beta$-sheet, it extends over the edge of this sheet to also contact the D strand, where it overlaps with the 2/6.14 epitope (Fig. 4A and E). Fab 2/1.12 has the most contact with four residues in $\beta$-strands A, B and E of D3 (Fig. 4F). The sidechains of Thr-508 from D3 $\beta$-strand A and His-528 from strand B form hydrogen bonds with the sidechain of Tyr-32 from light chain complementaritydetermining region 1 (L-CDR1). In adjacent interactions, Tyr-577 in $\beta$-strand $\mathrm{E}$ forms a sidechain hydrogen bond to the backbone of Gly-91 in L-CDR3 while the Tyr-577 backbone hydrogen bonds to the sidechain of Asn-104 in H-CDR3. Another close contact and backbone-backbone hydrogen bond between D3 E $\beta$-strand residue Ala575 and Tyr-102 from H-CDR3 further strengthens interaction (Fig 4F). All four of these major epitope residues are invariant among the species examined here (Fig. 4E), correlating with excellent cross-reactivity of $\mathrm{mAb}$ 2/1.12.

Although mAb 2/6.14 contacts residues in each $\beta$-strand of the D3 DCFG $\beta$-sheet, the major contacts are formed by Arg-565 in strand D and six residues in the F-G loop (Fig 4E and G). The F-G loop forms a large bulge on the D3 surface that fits into a cavity in the Fab centered between the three H chain CDR loops (Fig. 4D). The Arg-565 sidechain forms bidentate hydrogen bonds to the backbone of Tyr-91 in L-CDR3 and a cation-pi interaction with Tyr-32 in L-CDR1. All three Fab H chain CDR loops surround the D3 F-G loop with each CDR forming at least one of a total of four mainchain-mainchain hydrogen bonds. A network of two sidechainsidechain and two sidechain-backbone hydrogen bonds forms around FG loop residue Glu-603 and H-CDR2 residue Asn-56. Hydrogen bonds between Lys-597 and Ser-57 of H-CDR2 and salt bridge between Lys-602 and Asp-31 of H-CDR1 further extend the interaction with the F-G loop (Fig 4G). 
D3 contains six cysteine residues, all of which are conserved among Plasmodium species; however, only four of these form disulfide bonds (Fig. 4E and Fig. 5A). Cys-513 in the A-B loop forms a short-range disulfide to Cys-523 in the B strand that likely stabilizes the residues between Cys-513 and the beginning of the B strand, which are highly polar and mostly disordered. Cys-546 in the C strand and Cys-592 in the F strand form a longrange disulfide bond to bridge these two adjacent $\beta$-strands in the middle of the DCFG $\beta$-sheet. Two D3 cysteines are free. Free cysteines buried in hydrophobic regions are occasionally present in extracellular proteins. Cys-542 is well buried in the hydrophobic core. Cys-604 is exposed in D3-Fab complexes but in the full ectodomain may be buried by residues at the interface between D1 and D3, including residues N-terminal to D3 residue 502, which were not present in the crystallization construct (Fig. 5A and B).

HAP2 D3 in $P$. berghei is only 9 and $18 \%$ identical in sequence to $C$. reinhardtii and A. thaliana HAP2 D3, respectively; however, their three-dimensional structures are highly conserved (Fig. 5B). Their seven $\beta$ strands superimpose with very low root mean square deviations (Fig 5C). The long-range disulfide bond that connects the $\mathrm{C}$ and $\mathrm{F}$ strands is also conserved (Fig 5C). Both C. reinhardtii and $P$. berghei but not A. thaliana have long insertions in their A-B loops and a disulfide bond that may help to stabilize these long loops, which orient quite differently (Fig. 5C).

There is little variation in the orientation and length of the BC, DE and FG loops at the N-terminal end of D3. These loops are stabilized by an Asn in the BC loop, an Asp in the FG loop, and the hydrogen bonds they make. The Asn, residue 531 in P. berghei (asterisked in Fig. 5C), forms hydrogen bonds to both the BC and DE loops near the D1 junction (Fig. 5D and E). Invariance in the other two species, despite the evolutionary distance between the unicellular algae, the plant, and protozoan parasite, is of particular interest because Asn-531 is in an $\mathrm{N}$-glycosylation NX(S/T) sequon in $P$. berghei but its equivalents in the other species are not (Fig 5C). The two other species are known to N-glycosylated but Plasmodium species are not. In D3 constructs mutated to remove $\mathrm{N}$-linked sites, the $\mathrm{N}$ was left unchanged because it was much more conserved than the $(\mathrm{S} / \mathrm{T})$ position in other Plasmodium species. The B, D, and E strands that connect in these loops all contribute residues important in the $\mathrm{mAb} 2 / 1.12$ epitope (Fig. 5E).

To better understand the $P$. berghei HAP2 ectodomain and its binding to $2 / 6.14$ Fab, we obtained negative stain EM images and subjected $\sim 2,000$ particles to iterative alignment and classification. The $P$. berghei HAP2 ectodomain is rod-like with three or four oval densities arranged linearly along the rod (Fig. 4H panel 1 and Supplementary Fig. S4). The 2/6.14 Fab - ectodomain complex is L-shaped (Fig. 4H panel 2). Similar results were obtained for complexes with ectodomains with residues 43-617 and 61-611 (Supplementary Fig. S4). The two globules with stronger density correspond to the Fab, which has two domains per globule. The globules with weaker density correspond to the HAP2 ectodomain. Fab 2/6.14 binds essentially perpendicularly to one end of the HAP2 rod, in agreement with the crystal structure showing that the Fab binds to the side of D3 (Fig. 4A). When the putative Fab-D3 portion of the class average was isolated with a mask (Fig.4H panel 3), it correlated well (0.959) with the projection of the crystal structure of the 2/6.14 Fab-D3 complex (Fig. 4H panel 4), as shown with the ribbon cartoon in the same orientation (Fig. 4H panel 6). When the mask was omitted and the D3 Fab complex was cross-correlated with the entire ectodomain Fab complex, the same result and orientation was obtained, but the cross-correlation score was lower because the crystal structure lacks HAP2 D1 and D2.

\section{Differences in antibody reactivity with $\mathrm{D} 3$ and the ectodomain}

Because of their differences in blocking transmission, we wondered whether mAbs differed in reactivity with the HAP2 ectodomain. Yields of monomeric ectodomain were lower than for D3 as described in Methods; nonetheless, gel filtration yielded a sharp peak (Fig. 6A). The peak primarily contained a band at $75 \mathrm{kDa}$, in agreement with the expected size of the ectodomain; furthermore, its $\mathrm{C}$-terminus was present as shown by detection of the C-terminal His-tag by Western blotting (Fig. 6B). Treatment with Endo D reduced the size of the ectodomain to $\sim 71 \mathrm{kDa}$ (Fig. $6 \mathrm{~B}$ ).

Presence of D3 epitopes in the ectodomain was tested by immunoprecipitation. Immunoprecipitation of the ectodomain, and D3 as a control, was detected using Western blotting of the His tag (Fig. 6C). mAb 2/6.14 was fully reactive with the ectodomain as shown by immunoprecipitation comparable to that with the His-tag antibody. In contrast and surprisingly, the other four mAbs were only partially reactive with the ectodomain, although they reacted with D3 comparably to mAb 2/6.14. 
These differences were followed up by further comparisons that focused on mAbs 2/6.14 and 2/1.12. Calculations of affinity and dissociation constants assume fully active material; if only a fraction is active, then the apparent affinity is lower. In SPR, only the concentration of the analyte enters into affinity calculations. Therefore, we compared affinities measured for the two Fabs using them either as analyte or immobilized on the chip (Fig. 6E and Supplementary Fig. S1). For Fab 2/1.12, the ratio of $\mathrm{K}_{\mathrm{D}}$ values for D3 (analyte/immobile) was $5.2 / 5.9=0.88 \pm 0.09$ (Fig. $6 \mathrm{E}$ ). In contrast, these values for the ectodomain were $106 / 11.7=9.1 \pm 6.2$. Thus, the ectodomain appears to be only $0.88 / 9.1=9 \pm 6 \%$ active in binding to Fab $2 / 1.12$. Comparable $K_{D}$ value ratios for $\mathrm{Fab} 2 / 6.14$ were $6.0 / 10.2=0.59 \pm 0.23$ for $\mathrm{D} 3$ and $22.9 / 50.5=0.45 \pm 0.04$ for the ectodomain. Thus, the ectodomain appears to be $0.59 / 0.45=130 \pm 52 \%$ active in binding $\mathrm{mAb} 2 / 6.14$, within error of the expected value of $100 \%$, while Fab 2/1.12 binding to the ectodomain was far below the expected value.

We also assessed epitope content of the ectodomain by mixing it with equimolar concentrations of Fab and separating the complexes from components in gel filtration. Gel filtration of the mixtures was compared to that of the same concentrations of unmixed components (Fig. 6D). With Fab 2/6.14, much of the ectodomain was shifted to higher molecular weight and the Fab fragment was substantially depleted. With Fab 2/1.12 in contrast, only a small portion of the ectodomain was shifted to a higher molecular weight shoulder and there was little or no depletion of the Fab.

We further measured affinity with bio-layer interferometry (BLI) and complex formation with an independent preparation of the ectodomain. $\mathrm{mAb}$ IgG were immobilized on anti-mouse Fc capture sensors. HAP2 D3 bound well to both mAbs 2/6.14 and 2/1.12. In contrast, HAP2 ectodomain bound to mAb 2/6.14 but not to $\mathrm{mAb} 2 / 1.12$ (Supplementary Fig. S5). In conclusion, mAb 2/6.14 binds well to both D3 and the ectodomain, whereas $\mathrm{mAb} 2 / 1.12$ and three other antibodies bind well to the D3 immunogen but bind poorly to or do not recognize the ectodomain.

\section{DISCUSSION}

We have generated a mAb to HAP2 D3 that blocks Plasmodium ookinete formation in vitro and in vivo and have determined a crystal structure of D3 that reveals the epitope to which the neutralizing mAb binds. Furthermore, we found that a subset of D3 mAbs were not fully reactive with the HAP2 ectodomain and that these antibodies were ineffective in preventing ookinete conversion. These findings have important implications for choice of future HAP2 immunogens. Previously, in the absence of structural knowledge, a large number of $P$. berghei HAP2 cDNA clones beginning and ending in different positions were tested in E. coli. Only one sequence, residues 355-609, was found to be expressed well; fortunately, it yielded an immunogen that elicited polyclonal antisera that inhibited gamete fertilization in vitro and reduced transmission in vivo (9). This sequence contained about half of D2, a small portion of D1, and all of D3 except for the last four residues of strand G. Recently, polyclonal antibodies to the putative fusion loops of D2 of Plasmodium berghei HAP2 also showed efficacy in blocking fertilization (8). The same laboratory and procedures were used for these studies, so the results are directly comparable, except for the use of a polyclonal antibody in the previous and monoclonal antibody in the current study. Surprisingly, mAb 2/6.14 to D3 is at least 5-fold more effective than that affinitypurified polyclonal antibody, as $100 \mu \mathrm{g} / \mathrm{ml}$ reduced oocyst intensity by $78 \%$, whereas $500 \mu \mathrm{g} / \mathrm{ml}$ affinity-purified polyclonal antibodies to the fusion loop reduced intensity by $61 \%$. Recently, residues 231-459 of $P$. vivax HAP2 were also tested. It contains $\sim 30 \%$ of D1 and $\sim 60 \%$ of D2 and, as expected from lack of a complete domain, had to be recovered from inclusion bodies in insect cells (10). This material elicited antibodies that inhibited transmission, but the results cannot be directly compared. The present study is the first time that a defined domain from HAP2 has been used in immunization, that a monoclonal antibody has been found to be effective in blocking transmission, and that a Plasmodium species HAP2 structure has been reported. Thereby, this study advances the rational development of transmission-blocking malaria vaccines.

HAP2 is highly conserved, both within and between Plasmodium species. In P. falciparum, 199 isolates in PlasmoDB (www.plasmodb.org, release 54) evaluated have identical D3 amino acid sequences and only a few polymorphisms are present in D1 and D2 of HAP2 ectodomain in 2 or more isolates (R99E, n=2; N184S, n=14; $\mathrm{D} 185 \mathrm{Y} / \mathrm{E}, \mathrm{n}=11 / 7$ and $\mathrm{D} 455 \mathrm{~N}, \mathrm{n}=47$ ). The high sequence identity of gamete antigens contrasts to malaria vaccine antigens expressed by sporozoites such as TRAP and the C-terminal region of CSP used in the RTS,S vaccine and those expressed by blood stage parasites such as MSP1 and AMA1, which have high levels of polymorphisms 
and have proven to be challenging for vaccine antigen design (22-25). Compared to CSP and TRAP, HAP2 is also highly conserved among species; for example, P. berghei HAP2 is $60-70 \%$ identical in D3 to the five species we examined that are capable of infecting humans.

We structurally characterized HAP2 D3 in complex with two Fab fragments that bound to opposite faces of D3, on the $\beta$-sheets that form its $\beta$-sandwich domain. Conservation or variation among residues in these interfaces among Plasmodium species provides insights into which residues are important in their epitopes. The four residues most important for $\mathrm{mAb} 2 / 1.12$ binding were identical among all six species examined, explaining the wide cross-reactivity of this mAb. Although the D3 F-G loop was central in the 2/6.14 epitope, many of its contacts were between backbone atoms. The sidechain with the most contacts was Arg-565 in the D strand. All three interactions by the Arg-565 sidechain would be abolished by the Asn substitution in $P$. vivax and $P$. falciparum (Fig 4E), which had the lowest affinity.

Thus far, structural information on HAP2 has been on its post-fusion, trimeric form or on isolated domains (2-5). The rod-like, linear conformation of the HAP2 ectodomain and its monomeric state has not previously been seen for HAP2. Its resemblance to the linear conformation of pre-fusion states of structurally homologous class II viral fusogens suggests that this linear conformation of the HAP2 ectodomain corresponds to its pre-fusion state. The pre-fusion state is preferred over the post-fusion state as the target for transmission blocking antibodies, because antibodies that bind to the pre-fusion state not only can act earlier but can also sterically block the interfaces required for trimer formation and the fold-back of D3 onto D1 and D2 (Fig. 1).

In addition to immunological mechanisms for blocking gamete fertilization in the mosquito blood meal (11), mAb 2/6.14 might also block conformational changes required for conversion of the monomeric pre-fusion state of HAP2 to the trimeric post-fusion state (Fig 1), especially reorientation of D3 to fold over D1 and D2 in the trimer, which is required for fusion (Fig 1). Direct blocking of fusion has been demonstrated with antibodies to D3 of viral type II fusion proteins that are structurally homologous to HAP2 (13-15).

To test this idea, we superimposed D3 from $P$. berghei HAP2 on D3 from the trimeric fusion conformation of HAP2 in other species (Fig. 5B). The C. reinhardtii and A. thaliana D3 structures superimpose well, despite only 8 and $17 \%$ identity with $P$. berghei HAP2 D3, respectively. This identity is too low for immunological crossreactivity. Superposition showed that the F-G loop recognized by mAb 2/6.14 is buried in D3 interfaces in the postfusion state trimer. The mAb 2/1.12 epitope also substantially overlaps with the D3 postfusion state trimer interfaces in the $\mathrm{C}$ strand. Moreover, both antibody epitopes overlap in the $\mathrm{D}$ strand with sites buried in the postfusion conformation. The incompatibility of Fab binding and conformational change to the postfusion state is shown by burial of the Fabs in superpositions on one monomer in a postfusion trimer of $C$. reinhardtii HAP2 (Supplemental Fig. S6). Thus, binding of either of these mAbs to D3 would block folding back of $\mathrm{D} 3$ in the fusion state (Fig. 1C). Why then did mAb 2/6.14 and not mAb 2/1.12 block conversion of macrogametes to ookinetes?

$\mathrm{mAb} 2 / 6.14$ is differentiated from the other $4 \mathrm{mAbs}$ studied here by its ability to completely react with the monomeric HAP2 ectodomain. Although all five mAbs reacted with D3 with nanomolar EC50 values by ELISA and pulled down similar amounts of isolated D3 by immunoprecipitation, only mAb 2/6.14 fully immunoprecipitated the ectodomain. SPR measurements further showed that the affinity of mAb 2/1.12 was substantially lower for the ectodomain than for D3, but only when the ectodomain was used as analyte and not when immobilized on the chip, suggesting that only $\sim 10 \%$ of the ectodomain was active in binding to $\mathrm{mAb}$ 2/1.12. Gel filtration showed that the ectodomain complexed well with mAb $2 / 6.14$, whereas only a minor fraction of the ectodomain complexed with $\mathrm{mAb} 2 / 1.12$. Thus, three independent methods show that only a minor fraction of the HAP2 ectodomain reacts with $\mathrm{mAb} 2 / 1.12$, and one of these methods, immunoprecipitation, showed that another three $\mathrm{mAbs}$ behaved like $\mathrm{mAb} 2 / 1.12$. While all $5 \mathrm{mAbs}$ gave clear-cut staining of $P$. berghei microgametes, the microgametes had been fixed in $4 \%$ paraformaldehyde and incubated overnight in this solution, which denatures many proteins, prior to staining.

The scope of this study was to investigate the effectiveness of HAP2 D3 as an immunogen for transmission-blocking malaria vaccines. Nonetheless, our study also provides some useful guideposts for future use of the HAP2 ectodomain in transmission-blocking vaccines. Most eukaryotic extracellular proteins are highly dependent on N-glycosylation for stability and expression; however, lack of N-glycosylation sites in D3 appeared to have little or no effect on expression yield, which favors the hypothesis that N-glycans are not added to HAP2. 
Unfortunately, efficiently expressing eukaryotic extracellular proteins with complex multi-domain structures, multiple disulfide bonds, and no $\mathrm{N}$-glycosylation requires refolding from E. coli, which is highly challenging. Therefore, the best approach for successfully expressing the HAP2 ectodomain may be to use eukaryotic hosts and to mutationally remove N-glycosylation sequons, as was done here for D3 but not for the ectodomain.

In conclusion, we find that D3 of HAP2 can elicit antibodies that block transmission of malaria. Furthermore, we have obtained a mAb that blocks transmission, determined the first structure of a fragment of HAP2 in Plasmodium, and determined the structure of HAP2 of D3 in Plasmodium in complex with antibodies that either block or do not block transmission. We have confirmed the principle that HAP2 D3 can elicit transmission-blocking antibodies. On the other hand, we have also identified limitations of D3 as an immunogen because some antibodies to D3 did not react well with the HAP2 ectodomain. We also outline a possible strategy for obtaining improved expression and more native folding of the HAP2 ectodomain as an alternative immunogen for transmission-blocking immunity. This proposed approach is validated by our EM studies showing we can isolate a monomeric, pre-fusion state of the HAP2 ectodomain. Furthermore, stabilizing the pre-fusion state of HAP2, as successfully done for the respiratory syncytial virus fusion protein (26) and SARS-CoV-2 spike protein (27), may not only increase expression but also efficacy in inducing neutralizing antibodies. 


\section{MATERIALS AND METHODS}

Study design. This study was designed to provide insights for developing a vaccine to block or reduce malaria transmission. This objective was addressed by first conducting producing monoclonal antibodies against the D3 fragment of Plasmodium berghei HAP2. CB6F1 mice were immunized with the glycan-shaved D3 protein. All in vitro characterization of binding properties was carried out after a detailed antibodies screening as described in the associated figure legends. We next tested the ability of the $\mathrm{D} 3 \mathrm{mAbs}$ to inhibit $P$. berghei microgametes from fertilizing macrogametes and forming ookinetes in vitro using standard membrane feeding assay. The results show that all five antibodies react with microgametes, that $\mathrm{mAb} 2 / 6.14$ is outstanding for its ability to block $P$. berghei fertilization in vitro. Therefore, we decided to test $\mathrm{mAb} 2 / 6.14$ for its ability to block transmission in vivo in mosquitoes. For in vivo characterization of the ability of mAb $2 / 6.14$ to reduce malaria transmission, Female Anopheles stephensi mosquitoes were randomized to group feed through membranes on blood from $P$. berghei infected mice mixed with mAb 2/6.14 or control IgG. After 14 days, oocyst intensity and prevalence of infection mosquito were counted to evaluate the ability of mAb 2/6.14 inhibition.

Ethical statement for animal studies. Mouse immunization was conducted in accordance with and was approved by Boston Children's Hospital Institutional Animal Care and Use Committee (IACUC) under protocol \#14-06-2731. Animals were cared in compliance with the U.S. Department of Agriculture (USDA) Animal Welfare Act (AWA) and the Public Health Service (PHS) Policy on Humane Care and Use of Laboratory Animals.

Immunization and Plasmodium berghei infection procedures were performed in accordance with the UK Animals (Scientific Procedures) Act (PPL 70/8788) and were AWERB approved. The Office of Laboratory Animal Welfare Assurance covers all Public Health Service supported activities involving live vertebrates in the United States (no. A5634-01).

HAP2 constructs, expression and protein purification. $P$. berghei HAP2 amino acid sequence numbering is from RefSeq accession XP_022713330.1. D3 constructs utilized residues 477-621, 497-617, or 502617 with a C-terminal His tag; the 502-617 construct contained N516T, S533N and N539Q mutations to abolish N-glycosylation. HAP2 D3 construct sequence ranges and accessions in other species were P. falciparum, 479626, XP_001347424.1; P. knowlesi, 492-607, XP_002258781.1; P. vivax, 492-608, SGX77070.1; P. malariae, 498-613, SCN12177.1; and P. ovale, 493-608, SBS88209.1. These D3 constructs were expressed either transiently in Expi293F cells using pD2529-CAG vector (Atum, Newark, CA), or in the case of the P. berghei HAP2 D3 477-621 construct and ectodomain 61-611 and 43-617 constructs, and the P. falciparum D3 479-626 construct in Drosophila Schneider S2 cells, were expressed exactly as described previously (3). All constructs were codon-optimized for mammalian cells. UniProt accession Q4YCF6.1, which was used for P. berghei HAP2 ectodomain construct codon optimization, was retrospectively discovered to be deleted for S206, which locates to the $\beta \mathrm{d}$-strand of D2.2 in the alignment to Chlamydomonas reinhardti HAP2, at the opposite end of the ectodomain from D3. All other eight HAP2 accessions recovered from the nonredundant protein database with NCBI BLAST in 2020, including at least seven distinct $P$. berghei strains including ANKA, are identical to one another and differ from the Q4YCF6.1 sequence only at residue S206 and the signal sequence. This error has been reported to help@uniprot.org. Our recent experience is that, among hundreds of sequences of human and mouse extracellular proteins of similar length, $\sim 10 \%$ of UniProt but not RefSeq accessions have similar errors.

For purification, HAP2 fragments in $1 \mathrm{~L}$ of culture supernatant were adjusted to $1 \mathrm{mM} \mathrm{NiCl}_{2}$ in D3 buffer (20 mM Tris, $\mathrm{pH} 8$ and $300 \mathrm{mM} \mathrm{NaCl}$ ) or ectodomain buffer $(20 \mathrm{mM}$ Tris, $\mathrm{pH} 8.5,500 \mathrm{mM} \mathrm{NaCl})$ and applied to $10 \mathrm{ml}$ Ni-NTA-Sepharose (Qiagen) columns. After washing with $15 \mathrm{mM}$ imidazole in D3 or ectodomain buffer, the protein was eluted with $300 \mathrm{mM}$ imidazole in the same buffers. Fragments were then subjected to gel filtration chromatography using Superdex 75 (GE life Sciences) in D3 or ectodomain buffer, concentrated, and frozen at $80^{\circ} \mathrm{C}$. Purification of $P$. berghei 477-621 D3 construct expressed stably in S2 cells was similar, except after the Ni-NTA step it was concentrated $(1 \mathrm{mg} / \mathrm{ml}$ in $0.2 \mathrm{ml})$ and shaved with endoglycosidase D (10 $\mu 1,100$ unit) (New England BioLabs) for $16 \mathrm{hrs}$ at $4^{\circ} \mathrm{C}$ prior to gel filtration. Final yield was $22 \mathrm{mg} / \mathrm{L}$ culture supernatant. Yields for transiently expressed D3 constructs were $\sim 5$ to $8 \mathrm{mg} / \mathrm{L}$ culture supernatant. Ectodomain yields were $\sim 0.2-0.3$ $\mathrm{mg} / \mathrm{L}$ culture supernatant.

Monoclonal antibodies. CB6F1 mice (Charles River, Wilmington, MA) were immunized intraperitoneally with $20 \mu \mathrm{g}$ of N-glycan shaved P. berghei HAP2 D3 (residues 477-621) in PBS and complete 
Freund's adjuvant (Sigma) and 3 weeks later with the same material in incomplete Freund's adjuvant (Sigma). Mice were boosted both intravenously and intraperitoneally 2 weeks later with $20 \mu \mathrm{g}$ of the same antigen in PBS. Three days later, splenocytes were fused with the murine myeloma P3X63Ag8.653 (CRL 1580, ATCC, Rockville, MD) as described (28). Hybridomas supernatants were screened by ELISA in microtiter wells coated with the immunogen. Hybridomas that produced IgG mAbs were subcloned. Five produced antibodies specific for $P$. berghei HAP2 D3 and are characterized in Results.

$\mathrm{mAb} 1 / 5.13$ was found to be specific to the His-tag. It reacts with proteins with C-terminal His tags, but not with N-terminal His tags. It is fully active in ELISA, Western blot and immunoprecipitation and has sensitivity comparable to the $\mathrm{THE}^{\mathrm{TM}}$ His Tag Antibody (Genscript) in ELISA and Western Blot (Supplementary Fig.S7).

For antibody purification, hybridoma cells were adapted to and expanded in serum free medium containing 1:2 vol/vol Cell ${ }^{\mathrm{TM}}$ MAB medium (Life Technologies) and HyClone ${ }^{\mathrm{TM}}$ CDM4MAB medium (GE Life Sciences). IgG was purified using protein $\mathrm{G}$ affinity chromatography (Invitrogen).

Heavy and light chain $\mathrm{V}$ region cDNA sequences of mAbs 2/6.14 and 2/1.12 were determined by Syd Labs (Natick, MA). The mAbs each have $\gamma 1$ heavy and $\kappa$ light chains and unique $\mathrm{V}$ regions as shown by BLAST searches. A somatic mutation in the 2/6.14 VL domain that introduced an N-glycosylation site into the framework region was reversed with an N74S mutation (mature protein numbering) in recombinantly expressed Fab and IgG. Fab or intact $\mathrm{H}$ chains used g-Blocks encoding the murine $\kappa$ chain secretion signal peptide, $\mathrm{VH}$ and $\gamma 1 \mathrm{CH} 1$ domain, with or without hinge, $\mathrm{CH} 2$ and $\mathrm{CH} 3$ domains, followed by a Gly-Ser linker and 6xHis tag, that were assembled in EcoRV-linearized pVRC8400 vector (29) using NEBuilder ${ }^{\circledR}$ HiFi DNA reagents and protocol (New England BioLabs). $\kappa$ light chains used g-Blocks encoding the murine $\kappa$ chain secretion signal peptide, VL domain, and CL domain that were similarly assembled with SapI-linearized pD2529-CAG vector (Atum, Newark, CA).

Fabs and IgGs were expressed in Expi293F cells co-transfected with $\mathrm{H}$ and L chain constructs in 2:1 ratios. Fab fragments were purified from culture supernatant by Ni-NTA affinity purification followed by Superdex 200 gel filtration chromatography. IgG was purified by protein G affinity chromatography. 2/6.14 IgG purified from Expi293F and hybridoma cell supernatants bound to P.berghei HAP2 D3 in ELISA with comparable EC50 values.

Enzyme-linked immunosorbent assay (ELISA). 96-well ELISA plates (Costar) were coated overnight at $4^{\circ} \mathrm{C}$ with $50 \mu 1$ of purified, His-tagged HAP2 D3 at $5 \mu \mathrm{g} / \mathrm{ml}$ in $50 \mathrm{mM}$ sodium carbonate buffer, pH 9.5 and blocked with $3 \%$ BSA for $90 \mathrm{~min}$ at $37^{\circ} \mathrm{C} .50 \mu \mathrm{l}$ of diluted hybridoma supernatants or purified mAbs in triplicate were incubated for $2 \mathrm{hrs}$ at $37^{\circ} \mathrm{C}$. After 3 washes, $50 \mu \mathrm{l}$ of 1:10,000 diluted HRP-conjugated goat-anti-mouse IgG $(\mathrm{H}+\mathrm{L})($ Abcam) was added. After $1 \mathrm{~h}$ at room temperature and 4 washes, peroxidase substrate (Life Technologies) was added and after $10 \mathrm{~min}$ plates were read at $405 \mathrm{nM}$ on an Emax plate reader (Molecular Devices). As a control, hybridoma medium or mAb dilution buffer (1\% BSA in PBS) was substituted in the antibody binding step. For measuring binding of His-tagged D3 to immobilized mAbs, ELISA plates were coated with $5 \mu \mathrm{g} / \mathrm{ml}$ $\mathrm{mAb}$, and HRP-anti-His (Penta-His Ab, Qiagen) was used in the detection step. Titration curve fitting and EC50 measurements used the sigmoidal, 4 parameter logistic equation in GraphPad Prism 7.

Surface plasmon resonance (SPR). Purified HAP D3 (residues 502-617 with N516T, S533N and N539Q mutations) or ectodomain (residues 43-617) fragments or Fabs 2/1.12 or 2/6.14 were either used as analytes or amine immobilized on a CM5 chip in a Biacore 3000 (GE Healthcare) according to the manufacturer's instructions. For immobilization, protein was diluted to $5 \mu \mathrm{g} / \mathrm{ml}$ in $0.15 \mathrm{M} \mathrm{NaCl}, 20 \mathrm{mM}$ Hepes $\mathrm{pH} 7.4$, and injected at $10 \mu \mathrm{L} / \mathrm{min}$. The surface was regenerated with a 10- to 60 -s pulse of $18 \mathrm{mM} \mathrm{HCl}$ at the end of each cycle to restore resonance units to baseline. Kinetics and affinity analysis were performed with SPR evaluation software version 4.0.1 (GE Healthcare). A 1:1 Langmuir binding model, with or without a conformational change model, was applied for experimental data fitting, and kinetic parameters were fit globally to sensorgrams at different analyte concentrations.

Bio-layer interferometry (BLI). Bio-layer interferometry (30) experiments were performed on a ForteBio Octet RED384 instrument using anti-mouse Fc capture (AMC, 18-5090) sensors. The reaction was measured on a 384 well plate (working volume of $25 \mu \mathrm{L}$ ) in $20 \mathrm{mM}$ Tris- $\mathrm{HCl}, \mathrm{pH} 7.5,150 \mathrm{mM} \mathrm{NaCl}, 0.01 \% \mathrm{BSA}$, $0.02 \%$ Tween 20 (Assay buffer). Biosensors were hydrated in assay buffer for 10 min before starting the measurements. Each biosensor was sequentially moved through 5 wells with different components: (1) Assay 
buffer for 1 minute in baseline equilibration step; (2) $5 \mathrm{ug} / \mathrm{ml}(33.3 \mathrm{nM}) 2 / 6.14$ or 2/1.12 $\mathrm{IgG}$ for 3 minutes for immobilization of the antibodies onto the biosensor; (3) Assay buffer for 3 minutes for another baseline equilibration; (4) indicated concentrations of PbHAP2 ectodomain (aa:43-617) or PbD3(aa: 477-621) for 10 minutes for the association phase measurement; and (5) Assay buffer for 10 minutes for the dissociation phase measurement. Each biosensor has a corresponding assay buffer reference sensor that went through the same 5 steps. Kinetics and affinity analysis were performed with Octet RED384 Data Analysis 11.0. A 1:1 Langmuir binding model was applied for experimental data fitting, and kinetic parameters were fit globally to different analyte concentrations for each IgG and HAP2 combination, with $k_{\mathrm{on}}$ and $k_{\mathrm{off}}$ as shared fitting parameters and maximum response $\left(R_{\max }\right)$ as individual fitting parameter.

Immunoprecipitation and Western blot. Culture supernatants or purified HAP2 D3 and ectodomain fragments diluted in TBS $(25 \mathrm{mM}$ Tris, $\mathrm{pH} 8,300 \mathrm{mM} \mathrm{NaCl})$ containing $0.5 \%$ BSA were incubated with mAbs or control non-binding IgG overnight at $4^{\circ} \mathrm{C}$. Immunocomplexes were pulled down with protein $\mathrm{G}$ beads by incubation at $4^{\circ} \mathrm{C}$ for $2 \mathrm{hrs}$ with rotation. Beads were washed 3 times with $1 \mathrm{ml}$ TBS and bound proteins eluted in 1x Laemmli sample buffer containing 5\% $\beta$-mercaptoethanol and subjected to reducing SDS-polyacrylamide gel electrophoresis. Blots were probed with polyclonal rabbit anti-His $(0.4 \mu \mathrm{g} / \mathrm{ml}$, Cell Signaling), followed by incubation with HRP-conjugated goat-anti-rabbit (GE Healthcare) and chemiluminescence imaging using LAS4000 system (Fuji Film). Quantitation of protein bands used ImageJ software.

Assays with $\boldsymbol{P}$. berghei gametes. For immunofluorescent staining of microgametes, tail blood (10 $\mu$ l) from a mouse with high gametocytaemia was mixed with $10 \mu 1$ of ookinete medium (RPMI1640 containing $25 \mathrm{mM}$ HEPES, $20 \% \mathrm{FCS}, 100 \mu \mathrm{M}$ xanthurenic acid $\mathrm{pH}$ 7.4) to stimulate emergence of male gametes (exflagellation), which was monitored microscopically as described (31). Exflagellating gametocytes were fixed in solution for $15 \mathrm{~min}$ by diluting formalin to $4 \%$ paraformaldehyde, then added to poly-L-lysine-coated slides and incubated at $4^{\circ} \mathrm{C}$ overnight. For staining, mAbs or control mouse IgG UPC10 (M7769, Sigma Aldrich) $(2 \mu \mathrm{g}$ in $4 \mu 1$ of 3\% BSA/PBS) were added to washed slides; rabbit anti-alpha tubulin (Abcam AB18251) was at 1:1000 dilution. Following washes, Alex Fluor-488 anti-mouse IgG and Alexa Fluor-594 anti-rabbit IgG secondary antibodies (Molecular Probes) were added at $25 \mu \mathrm{g} / \mathrm{ml}$ before mounting in VectaShield ${ }^{\circledR}$ with DAPI (Vector Laboratories). Fluorescence images were obtained using an epifluorescence 100x objective on a Nikon Eclipse Ti microscope. Image handling used Adobe Photoshop CC.

In vitro ookinete conversion was assayed as described $(8,32)$. Briefly, infected mouse blood containing $P$. berghei (strain ANKA 2.34) female and male gametocytes (20 ul) was mixed with ookinete medium (100 ul) containing HAP2 D3 antibodies or control $\mathrm{IgG}\left(500\right.$ and $250 \mathrm{ug} / \mathrm{ml}$ final concentration) and incubated at $19^{\circ} \mathrm{C}$. After $24 \mathrm{~h}$, cultures were incubated with Cy3-conjugated Pbs $28 \mathrm{mAb}$ 13.1, which stains both ookinetes and unfertilized macrogametes (33d5904), for 20 min on ice. Larger, elongated, banana-shaped ookinetes were distinguished from smaller, round macrogametes and counted by fluorescence microscopy. Conversion rates were calculated as \% ookinetes /(macrogametes + ookinetes). Inhibition of ookinete conversion was expressed as the percentage reduction in ookinete conversion with respect to the negative control IgG at the same concentration.

In vivo transmission-blocking activity of HAP2 antibodies was assayed using standard membrane feeding assay (SMFA) as described (32). Briefly, heparinized $P$. berghei infected blood containing gametocytes was mixed with HAP2 antibodies or negative control IgG. Female Anopheles stephensi (SDA 500 strain) were starved for 24 hours and then fed on the mixtures using membrane feeders ( $>50$ mosquitoes per each blood-antibody feed). 24 hours later, unfed mosquitoes were removed. Mosquitoes were maintained on $8 \%(\mathrm{w} / \mathrm{v})$ fructose, $0.05 \%$ (w/v) p-aminobenzoic acid at $19-22^{\circ} \mathrm{C}$ and $50-80 \%$ relative humidity. Day 14 post-feeding, mosquito midguts were dissected, and oocyst numbers per midgut in each mosquito was determined by phase contrast microscopy. Reductions in oocyst intensity (number of oocysts/midgut) and prevalence (number of infected over total mosquitoes fed) in the presence of an HAP2 antibody were calculated with respect to the negative control $\operatorname{IgG}$ present at the same concentration in the feeds.

Crystallization and structure determination. The $P$. berghei D3 (502-617) construct with N516T, S533N and N539Q mutations to abolish N-glycosylation was used for crystallization. D3 and Fab were mixed in 1:1.3 molar ratios and complexes were isolated by gel filtration. Complexes were crystallized at $20^{\circ} \mathrm{C}$ by hangingdrop vapor diffusion with equal volumes of complex and well solution. The Fab 2/1.12 complex (4.5 mg/ml) was crystallized with $0.2 \mathrm{M}$ ammonium sulfate, 25\% PEG 3350, 0.1 M Bis-Tris pH5.5. The 2/6.14 Fab complex (7.5 
$\mathrm{mg} / \mathrm{ml}$ ) was crystallized with $0.3 \mathrm{M}$ proline, 22\% PEG 1500, 0.1 M HEPES pH7.5; crystals were dehydrated by soaking in solutions that had the starting concentrations of components in the protein and reservoir solutions while raising the concentration of PEG 1500 to 31\% in 3\% steps. Crystals of Fab 2/1.12-D3 and Fab 2/6.14-D3 were cryo-protected with reservoir solution containing $15 \%$ glycerol or $15 \%$ ethylene glycerol, respectively, in 2 steps of 5 and 10\% increase and plunged in liquid nitrogen. Data were collected at 100K on GM/CA beamline 23IDB at the Advanced Photon Source (Argonne National Laboratory) and processed with XDS (34). Structures were refined with PHENIX, built with Coot (35) and validated with MolProbity (36). Figures were made with PyMol.

The 2/1.12 Fab D3 complex structure was solved by molecular replacement in the Phenix suite (37) using a Fab search model (PDB ID 2A6D). The 2/6.14 Fab-D3 complex structure was solved by molecular replacement using D3 from the 2/1.12 Fab complex, the H chain of PDB ID 1IGC, and the L chain of PDB ID 5AZ2. Each crystal structure has two complexes in the asymmetric unit.

During model building and refinement of the 2/6.14 Fab complex, the Fab constant domains of one complex had good density, but the variable domains and D3 had broad but continuous density that was difficult to trace. In contrast, all domains of the other complex were easily traced. Furthermore, refinement remained stuck. Alternative space groups including those with lower symmetry or use of twin rules provided no improvement. We then realized that in the troublesome D3-Fab complex in the asymmetric unit, two alternative conformations were present for D3, VH, and VL, whereas $\mathrm{CH} 1$ and CL had a single conformation. The transition between dual and single conformations occurred at the elbows between VH and CH1 and between VL and CL; i.e. the two conformations differed in elbow angle. Further refinement, which largely treated each of the dual conformations of D3, VH1, and VL as rigid bodies based on their structure in the single conformation of the other D3-Fab complex, resulted in drop to the final $\mathrm{R}_{\text {work }}$ and $\mathrm{R}_{\text {free }}$ of 25.3 and $29.2 \%$, respectively.

The alternate conformations allow the VL domain in chain B and the D3 domain in chain C to pack against alternate conformation symmetry mates in different complexes along adjacent edges of the unit cell. Symmetry mates with the B conformation severely clash, while those with the distinct conformations A and B have good lattice contacts. In contrast, symmetry mates with conformation A are too far apart to provide stabilizing lattice contacts. Thus, clashes in one conformation together with a lack of stabilizing lattice contacts in the other conformation may have driven the formation of a crystal lattice with dual conformations of VL, VH, and D3 in one of two Fab-D3 complexes in the asymmetric unit.

Negative stain electron microscopy. Each P. berghei HAP2 ectodomain construct (residues 43-617 or 61-611), with or without Fab at a molar ratio of 1:1.3, was subjected to Superdex 200 gel filtration in $20 \mathrm{mM}$ Tris$\mathrm{HCl}, \mathrm{pH} 8,500 \mathrm{mM} \mathrm{NaCl}$. Peak fractions were adsorbed to glow-discharged carbon-coated copper grids, washed with deionized water, and stained with freshly prepared $0.75 \%$ uranyl formate. Images were acquired with an FEI Tecnai-T12 transmission electron microscope at $120 \mathrm{kV}$ and a nominal magnification of 52,000×. About two thousand particles were picked interactively and subjected to $2 \mathrm{D}$ alignment, classification and averaging using RELION-2.1 (38). Selected EM class averages were masked and cross-correlated using EMAN2 (39) with 2D projections generated at $2^{\circ}$ intervals from the $2 / 6.14$ Fab-D3 complex crystal structure filtered to $25 \AA$.

\section{Supplementary Materials}

Fig. S1. Surface plasmon resonance (SPR) analysis of binding Interactions of Fab 2/6.14 and Fab 2/1.12 with PbHAP2 D3 and monomeric ectodomain.

Fig. S2. Titration of binding of HAP2 D3 from Plasmodium species to immobilized mAbs.

Fig. S3. Transmission blocking activity of $\mathrm{mAb} 2 / 6.14$ in standard membrane feeding assay.

Fig. S4. EM class averages.

Fig. S5. Bio-layer interferometry (BLI) analysis of binding Interactions of IgG 2/6.14 and IgG 2/1.12 with PbHAP2 D3 and monomeric ectodomain.

Fig. S6. Analysis the mAb 2/6.14 and 2/1.12 binding in the context of postfusion C. reinhardtii HAP2 trimer. Fig. S7. Reactivity of $\mathrm{mAb} 1 / 5.13$ to fusion proteins with His tags.

Table S1. Statistics of X-ray diffraction and structure refinement of PbHAP2 domain 3 (D3) complexed with 2/6.14 Fab or $2 / 1.12 \mathrm{Fab}$ 


\section{References and Notes}

1. Clark, T. (2018) HAP2/GCS1: Mounting evidence of our true biological EVE? PLoS Biol 16, e3000007

2. Fedry, J., Liu, Y., Pehau-Arnaudet, G., Pei, J., Li, W., Tortorici, M. A., Traincard, F., Meola, A., Bricogne, G., Grishin, N. V., Snell, W. J., Rey, F. A., and Krey, T. (2017) The Ancient Gamete Fusogen HAP2 Is a Eukaryotic Class II Fusion Protein. Cell 168, 904-915 e910

3. Feng, J., Dong, X., Pinello, J., Zhang, J., Lu, C., Iacob, R. E., Engen, J. R., Snell, W. J., and Springer, T. A. (2018) Fusion surface structure, function, and dynamics of gamete fusogen HAP2. Elife 7

4. Fedry, J., Forcina, J., Legrand, P., Pehau-Arnaudet, G., Haouz, A., Johnson, M., Rey, F. A., and Krey, T. (2018) Evolutionary diversification of the HAP2 membrane insertion motifs to drive gamete fusion across eukaryotes. PLoS Biol 16, e2006357

5. Baquero, E., Fedry, J., Legrand, P., Krey, T., and Rey, F. A. (2018) Species-Specific Functional Regions of the Green Alga Gamete Fusion Protein HAP2 Revealed by Structural Studies. Structure

6. Hirai, M., Arai, M., Mori, T., Miyagishima, S. Y., Kawai, S., Kita, K., Kuroiwa, T., Terenius, O., and Matsuoka, H. (2008) Male fertility of malaria parasites is determined by GCS1, a plant-type reproduction factor. Curr Biol 18, 607-613

7. Liu, Y., Tewari, R., Ning, J., Blagborough, A. M., Garbom, S., Pei, J., Grishin, N. V., Steele, R. E., Sinden, R. E., Snell, W. J., and Billker, O. (2008) The conserved plant sterility gene HAP2 functions after attachment of fusogenic membranes in Chlamydomonas and Plasmodium gametes. Genes Dev 22, 10511068

8. Angrisano, F., Sala, K. A., Da, D. F., Liu, Y., Pei, J., Grishin, N. V., Snell, W. J., and Blagborough, A. M. (2017) Targeting the Conserved Fusion Loop of HAP2 Inhibits the Transmission of Plasmodium berghei and falciparum. Cell Rep 21, 2868-2878

9. Blagborough, A. M., and Sinden, R. E. (2009) Plasmodium berghei HAP2 induces strong malaria transmission-blocking immunity in vivo and in vitro. Vaccine 27, 5187-5194

10. Qiu, Y., Zhao, Y., Liu, F., Ye, B., Zhao, Z., Thongpoon, S., Roobsoong, W., Sattabongkot, J., Cui, L., Fan, Q., and Cao, Y. (2020) Evaluation of Plasmodium vivax HAP2 as a transmission-blocking vaccine candidate. Vaccine 38, 2841-2848

11. Graves, P. M., Carter, R., Burkot, T. R., Rener, J., Kaushal, D. C., and Williams, J. L. (1985) Effects of transmission-blocking monoclonal antibodies on different isolates of Plasmodium falciparum. Infect Immun 48, 611-616

12. Kielian, M., and Rey, F. A. (2006) Virus membrane-fusion proteins: more than one way to make a hairpin. Nat Rev Microbiol 4, 67-76

13. Austin, S. K., Dowd, K. A., Shrestha, B., Nelson, C. A., Edeling, M. A., Johnson, S., Pierson, T. C., Diamond, M. S., and Fremont, D. H. (2012) Structural basis of differential neutralization of DENV-1 genotypes by an antibody that recognizes a cryptic epitope. PLoS Pathog 8, e1002930 
14. Zhao, H., Fernandez, E., Dowd, K. A., Speer, S. D., Platt, D. J., Gorman, M. J., Govero, J., Nelson, C. A., Pierson, T. C., Diamond, M. S., and Fremont, D. H. (2016) Structural Basis of Zika Virus-Specific Antibody Protection. Cell 166, 1016-1027

15. Li, J., Watterson, D., Chang, C. W., Che, X. Y., Li, X. Q., Ericsson, D. J., Qiu, L. W., Cai, J. P., Chen, J., Fry, S. R., Cheung, S. T. M., Cooper, M. A., Young, P. R., and Kobe, B. (2018) Structural and Functional Characterization of a Cross-Reactive Dengue Virus Neutralizing Antibody that Recognizes a Cryptic Epitope. Structure 26, 51-59 e54

16. Delves, M. J., Angrisano, F., and Blagborough, A. M. (2018) Antimalarial Transmission-Blocking Interventions: Past, Present, and Future. Trends Parasitol 34, 735-746

17. Bushkin, G. G., Ratner, D. M., Cui, J., Banerjee, S., Duraisingh, M. T., Jennings, C. V., Dvorin, J. D., Gubbels, M. J., Robertson, S. D., Steffen, M., O'Keefe, B. R., Robbins, P. W., and Samuelson, J. (2010) Suggestive evidence for Darwinian Selection against asparagine-linked glycans of Plasmodium falciparum and Toxoplasma gondii. Eukaryot Cell 9, 228-241

18. Macedo, C. S., Schwarz, R. T., Todeschini, A. R., Previato, J. O., and Mendonca-Previato, L. (2010) Overlooked post-translational modifications of proteins in Plasmodium falciparum: N- and Oglycosylation -- a review. Mem Inst Oswaldo Cruz 105, 949-956

19. Bandini, G., Albuquerque-Wendt, A., Hegermann, J., Samuelson, J., and Routier, F. H. (2019) Protein Oand C-Glycosylation pathways in Toxoplasma gondii and Plasmodium falciparum. Parasitology 146, $1755-1766$

20. Swearingen, K. E., Lindner, S. E., Shi, L., Shears, M. J., Harupa, A., Hopp, C. S., Vaughan, A. M., Springer, T. A., Moritz, R. L., Kappe, S. H., and Sinnis, P. (2016) Interrogating the Plasmodium Sporozoite Surface: Identification of Surface-Exposed Proteins and Demonstration of Glycosylation on CSP and TRAP by Mass Spectrometry-Based Proteomics. PLoS Pathogens 12, e1005606

21. Swearingen, K. E., Eng, J. K., Shteynberg, D., Vigdorovich, V., Springer, T. A., Mendoza, L., Sather, D. N., Deutsch, E. W., Kappe, S. H. I., and Moritz, R. L. (2019) A Tandem Mass Spectrometry Sequence Database Search Method for Identification of O-Fucosylated Proteins by Mass Spectrometry. J Proteome Res 18, 652-663

22. Neafsey, D. E., Juraska, M., Bedford, T., Benkeser, D., Valim, C., Griggs, A., Lievens, M., Abdulla, S., Adjei, S., Agbenyega, T., Agnandji, S. T., Aide, P., Anderson, S., Ansong, D., Aponte, J. J., Asante, K. P., Bejon, P., Birkett, A. J., Bruls, M., Connolly, K. M., D'Alessandro, U., Dobano, C., Gesase, S., Greenwood, B., Grimsby, J., Tinto, H., Hamel, M. J., Hoffman, I., Kamthunzi, P., Kariuki, S., Kremsner, P. G., Leach, A., Lell, B., Lennon, N. J., Lusingu, J., Marsh, K., Martinson, F., Molel, J. T., Moss, E. L., Njuguna, P., Ockenhouse, C. F., Ogutu, B. R., Otieno, W., Otieno, L., Otieno, K., Owusu-Agyei, S., Park, D. J., Pelle, K., Robbins, D., Russ, C., Ryan, E. M., Sacarlal, J., Sogoloff, B., Sorgho, H., Tanner, M., Theander, T., Valea, I., Volkman, S. K., Yu, Q., Lapierre, D., Birren, B. W., Gilbert, P. B., and Wirth, D. F. (2015) Genetic Diversity and Protective Efficacy of the RTS,S/AS01 Malaria Vaccine. N Engl J Med 373, 2025-2037

23. Takala, S. L., Coulibaly, D., Thera, M. A., Batchelor, A. H., Cummings, M. P., Escalante, A. A., Ouattara, A., Traore, K., Niangaly, A., Djimde, A. A., Doumbo, O. K., and Plowe, C. V. (2009) Extreme polymorphism in a vaccine antigen and risk of clinical malaria: implications for vaccine development. $S c i$ Transl Med 1, 2ra5 
24. Barry, A. E., and Arnott, A. (2014) Strategies for designing and monitoring malaria vaccines targeting diverse antigens. Front Immunol 5, 359

25. Ouattara, A., Barry, A. E., Dutta, S., Remarque, E. J., Beeson, J. G., and Plowe, C. V. (2015) Designing malaria vaccines to circumvent antigen variability. Vaccine 33, 7506-7512

26. McLellan, J. S., Chen, M., Joyce, M. G., Sastry, M., Stewart-Jones, G. B., Yang, Y., Zhang, B., Chen, L., Srivatsan, S., Zheng, A., Zhou, T., Graepel, K. W., Kumar, A., Moin, S., Boyington, J. C., Chuang, G. Y., Soto, C., Baxa, U., Bakker, A. Q., Spits, H., Beaumont, T., Zheng, Z., Xia, N., Ko, S. Y., Todd, J. P., Rao, S., Graham, B. S., and Kwong, P. D. (2013) Structure-based design of a fusion glycoprotein vaccine for respiratory syncytial virus. Science 342, 592-598

27. Hsieh, C. L., Goldsmith, J. A., Schaub, J. M., DiVenere, A. M., Kuo, H. C., Javanmardi, K., Le, K. C., Wrapp, D., Lee, A. G., Liu, Y., Chou, C. W., Byrne, P. O., Hjorth, C. K., Johnson, N. V., Ludes-Meyers, J., Nguyen, A. W., Park, J., Wang, N., Amengor, D., Lavinder, J. J., Ippolito, G. C., Maynard, J. A., Finkelstein, I. J., and McLellan, J. S. (2020) Structure-based design of prefusion-stabilized SARS-CoV-2 spikes. Science 369, 1501-1505

28. Springer, T. A. (1980) Cell-surface differentiation in the mouse. Characterization of "jumping" and "lineage" antigens using xenogeneic rat monoclonal antibodies. in Monoclonal antibodies (Kennett, R. H., McKearn, T. J., and Bechtol, K. B. eds.), Plenum Press, New York. pp 185-217

29. Barouch, D. H., Yang, Z. Y., Kong, W. P., Korioth-Schmitz, B., Sumida, S. M., Truitt, D. M., Kishko, M. G., Arthur, J. C., Miura, A., Mascola, J. R., Letvin, N. L., and Nabel, G. J. (2005) A human T-cell leukemia virus type 1 regulatory element enhances the immunogenicity of human immunodeficiency virus type 1 DNA vaccines in mice and nonhuman primates. $J$ Virol 79, 8828-8834

30. Wallner, J., Lhota, G., Jeschek, D., Mader, A., and Vorauer-Uhl, K. (2013) Application of Bio-Layer Interferometry for the analysis of protein/liposome interactions. J Pharm Biomed Anal 72, 150-154

31. Sebastian, S., Brochet, M., Collins, M. O., Schwach, F., Jones, M. L., Goulding, D., Rayner, J. C., Choudhary, J. S., and Billker, O. (2012) A Plasmodium calcium-dependent protein kinase controls zygote development and transmission by translationally activating repressed mRNAs. Cell Host Microbe 12, 919

32. Blagborough, A. M., Delves, M. J., Ramakrishnan, C., Lal, K., Butcher, G., and Sinden, R. E. (2013) Assessing transmission blockade in Plasmodium spp. Methods Mol Biol 923, 577-600

33. Winger, L. A., Tirawanchai, N., Nicholas, J., Carter, H. E., Smith, J. E., and Sinden, R. E. (1988) Ookinete antigens of Plasmodium berghei. Appearance on the zygote surface of an $\mathrm{Mr} 21 \mathrm{kD}$ determinant identified by transmission-blocking monoclonal antibodies. Parasite Immunol 10, 193-207

34. Kabsch, W. (2001) F, Crystallography of Biological Macromolecules. in International Tables for Crystallography (Rossmann, M. G., and Arnold, E. eds.), Dordrecht: Kluwer Academic Publishers. pp 730-734

35. Emsley, P., and Cowtan, K. (2004) Coot: model-building tools for molecular graphics. Acta Crystallogr. D Biol. Crystallogr. 60, 2126-2132 
36. Davis, I. W., Leaver-Fay, A., Chen, V. B., Block, J. N., Kapral, G. J., Wang, X., Murray, L. W., Arendall, W. B., 3rd, Snoeyink, J., Richardson, J. S., and Richardson, D. C. (2007) MolProbity: all-atom contacts and structure validation for proteins and nucleic acids. Nucleic Acids Res. 35, W375-383

37. Adams, P. D., Afonine, P. V., Bunkoczi, G., Chen, V. B., Davis, I. W., Echols, N., Headd, J. J., Hung, L. W., Kapral, G. J., Grosse-Kunstleve, R. W., McCoy, A. J., Moriarty, N. W., Oeffner, R., Read, R. J., Richardson, D. C., Richardson, J. S., Terwilliger, T. C., and Zwart, P. H. (2010) PHENIX: a comprehensive Python-based system for macromolecular structure solution. Acta Crystallogr D Biol Crystallogr 66, 213-221

38. Zivanov, J., Nakane, T., Forsberg, B. O., Kimanius, D., Hagen, W. J., Lindahl, E., and Scheres, S. H. (2018) New tools for automated high-resolution cryo-EM structure determination in RELION-3. Elife 7

39. Ludtke, S. J., Baldwin, P. R., and Chiu, W. (1999) EMAN: semiautomated software for high-resolution single-particle reconstructions. J Struct Biol 128, 82-97

40. Liao, M., Sanchez-San Martin, C., Zheng, A., and Kielian, M. (2010) In vitro reconstitution reveals key intermediate states of trimer formation by the dengue virus membrane fusion protein. $J$ Virol 84, 57305740

41. Wang, S., Ma, J., Peng, J., and Xu, J. (2013) Protein structure alignment beyond spatial proximity. Sci $\operatorname{Rep~3,1448}$

42. Krissinel, E., and Henrick, K. (2007) Inference of macromolecular assemblies from crystalline state. $J$ Mol Biol 372, 774-797 
Acknowledgements. We thank Margaret Nielsen for illustrations. We thank Kelly L. Arnett from Center for Macromolecular Interactions of Harvard Medical school for training and consultation on Bio-layer

interferometry measurement. This work was supported by NIH grant 5R01AI95686 (T.A.S. and C.L.) the Kidder Fund from Boston Children's Hospital (T.A.S.), Medical Research Council grant MR/N00227X/1, Isaac Newton Trust, Alborada Fund, Wellcome Trust ISSF, University of Cambridge JRG Scheme, GHIT, Rosetrees Trust and the Royal Society (A.M.B.).

Author Contributions. J.F. contributed to experimental design, carried out the biochemical studies, HAP2 constructs and expression, negative stain EM, crystallization, solved the structure, and drafted the MS; X.D. contributed to finding and refining the dual conformations of the Fab 2/6.14 structure; A.D. contributed to Fab and HAP2 constructs and expression; F.A., K.A.S. and A.M.B. carried out Plasmodium berghei infection experiments and drafted the MS; Y.S. contributed to EM cross-correlation; C.L. generated and characterized monoclonal antibodies and constructs, and drafted the MS; C.L., A.M.B, and T.A.S. designed and supervised experiments and wrote the paper.

Competing interests. The authors declare no competing interests.

Data and materials availability. Protein database accession IDs are 7LR3 for 2/6.14- $\mathrm{Pb}$ HAP2 D3 complex and 7LR4 for 2/1.12- $P b$ HAP2 D3 complex. Correspondence and requests for materials should be addressed to CL and TAS. 


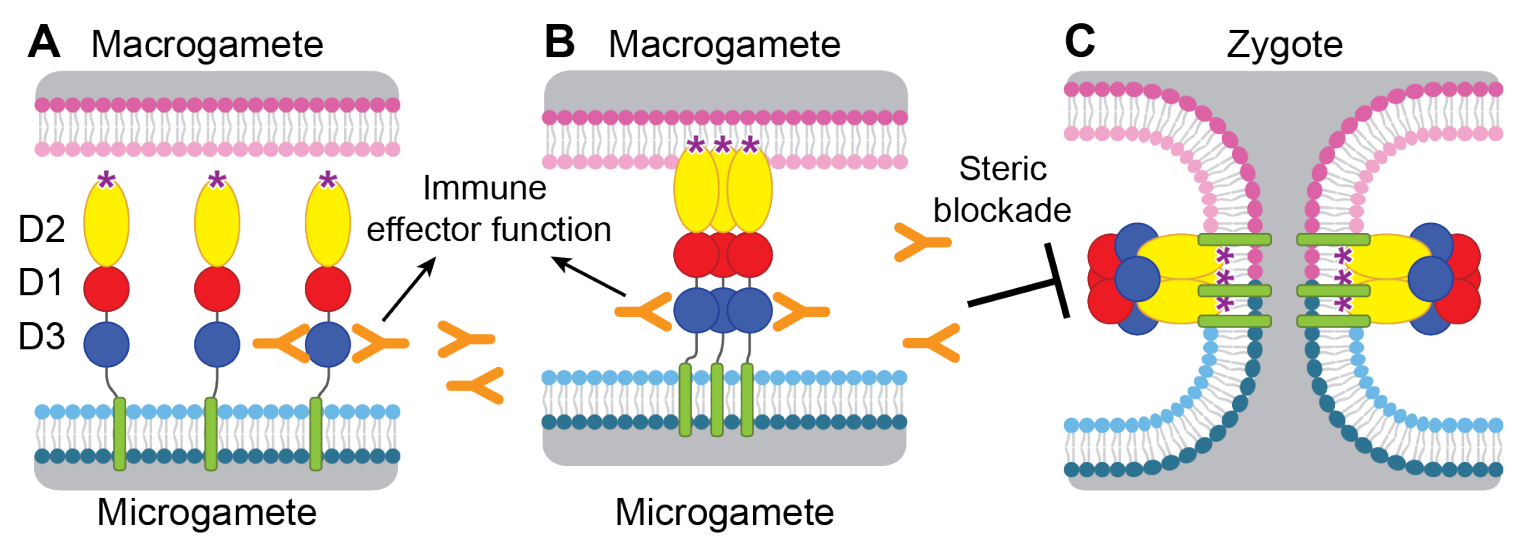

Fig. 1. Diagram of HAP2 pre-fusion monomer, trimerization and membrane fusion. The model is based on postfusion structures of HAP2 referenced in the text, their similarity to postfusion structures of viral class II proteins, models for the conversion of prefusion to postfusion class II fusion proteins (12), and work showing that D1 and D2 are sufficient for trimer formation (40) which suggests that the last step may be concerted foldback of D3 over D1 and D2 and fusion of the macrogamete and microgamete plasma membranes . (A) pre-fusion monomer on the surface of male gametes, (B) extended trimeric intermediate, and (C) post-fusion timer and fusion of gamete membranes. Domains are colored. Fusion loops at the tip of D2 are shown as “*”. Antibodies to D3, shown as Y shapes, may agglutinate microgametes, trigger antibody Fc-dependent effector functions, or neutralize HAP2 function by interfering with trimer formation and gamete membrane fusion. 

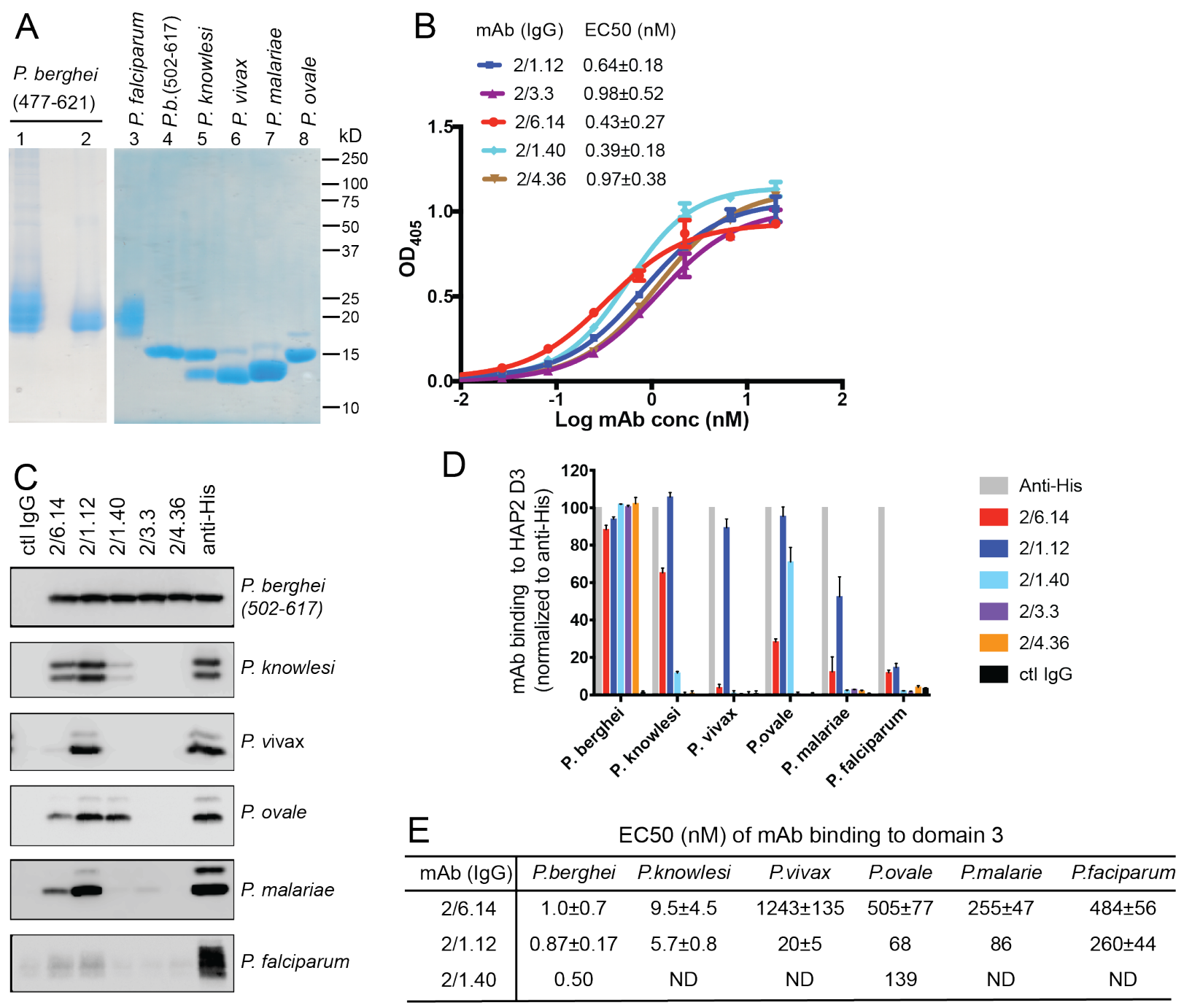

Fig. 2. Cross-reactivity of HAP D3 mAbs among Plasmodium species. (A) Reducing SDS 12.5\% PAGE of purified HAP2 D3 stained with Coomassie blue. Lanes 1 and 2: P. berghei HAP2 D3 (aa 477-621) purified by Ni-NTA (lane 1) and then treated with Endo D and purified by gel filtration (lane 2). Lanes 38: Purified HAP2 D3 fragments from Plasmodium species treated with Endo D or from P. berghei (aa 502-617) with N-linked sites removed by mutation. (B) Titration of antibody binding to Endo D-treated $P$. berghei HAP2 D3 (aa 477-621) by ELISA. Sigmoidal curve fits show one representative experiment with mean \pm difference from the mean of duplicates; EC50 values show mean \pm SD of 3 experiments. (C) Cross-species reactivity of HAP D3 mAbs by immunoprecipitation. Purified D3 proteins shown in (A) (2 ug each) were subjected to immunoprecipitation with the indicated mAbs, anti-His $1 / 5.13$, or control $\operatorname{IgG}$ (8 ug each). Immunocomplexes were analyzed by reducing $12.5 \%$ SDS-PAGE and Western blot using rabbit polyclonal antibodies to the C-terminal His tag of D3. (D) Quantitation of results from (C) and a repeat experiment. Intensities of bands were quantitated and data normalized to anti-His. Results shown are averages of the two experiments \pm difference from the mean. (E) EC50 measurements of HAP2 D3 binding to immobilized $\mathrm{mAbs}$ by Elisa. Purified D3 proteins shown in (A), lanes 3-8, were used. Sigmoidal curve fitting of D3 titration (Fig S2) and EC50 used GraphPad Prism 7 software. EC50 shown are mean \pm difference from means of two experiments, each experiment with triplicates. ND, Not determined. 


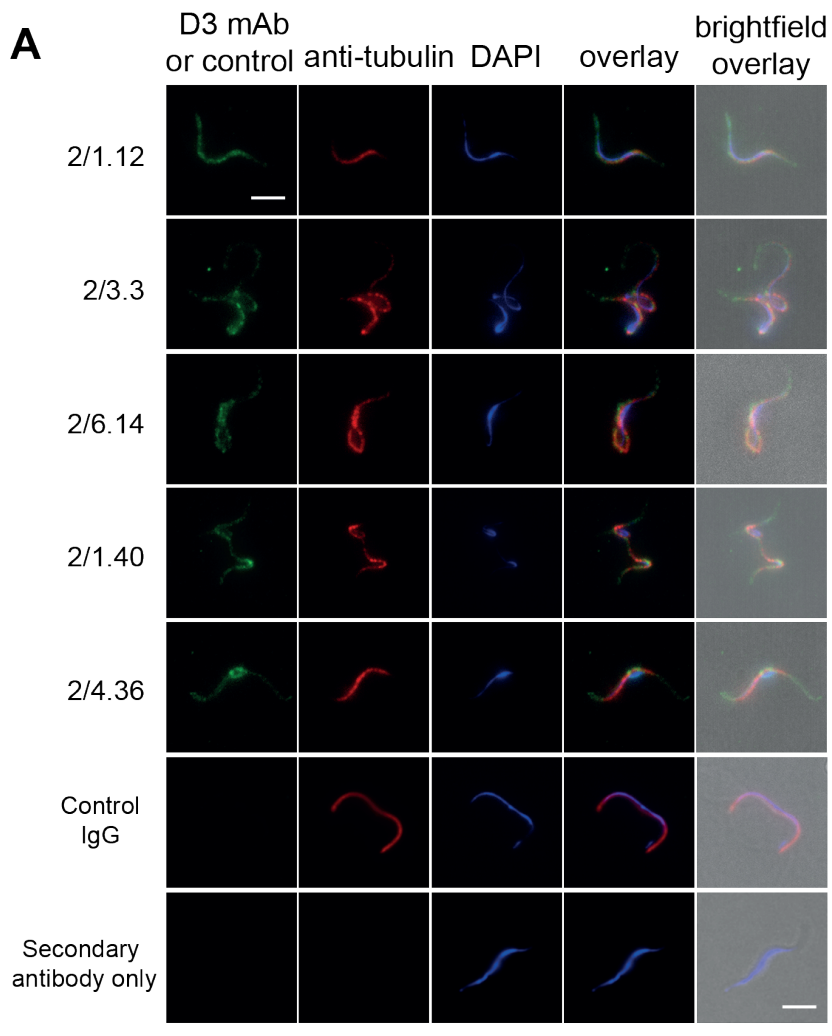

\section{B}

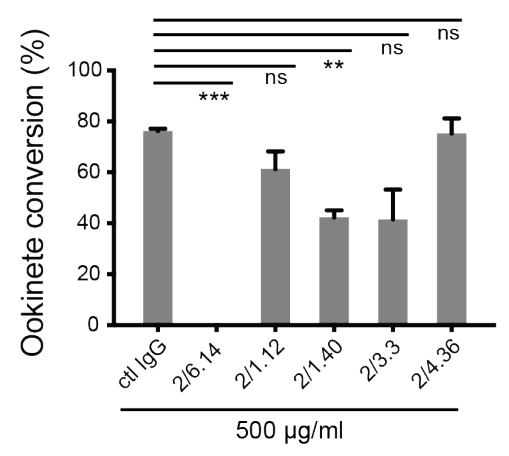

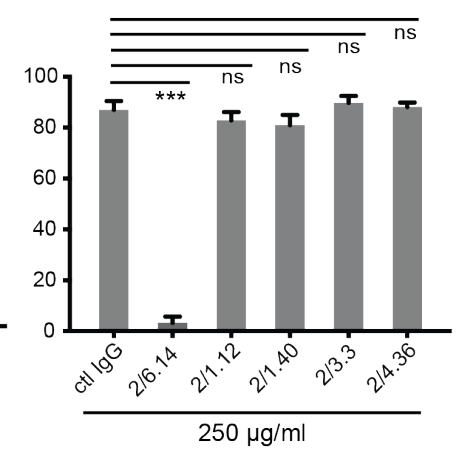

C

Expt 1

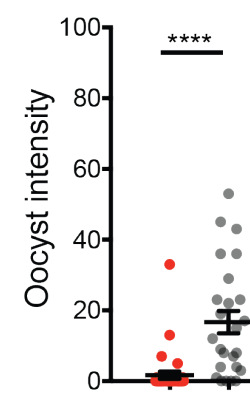

Expt 2

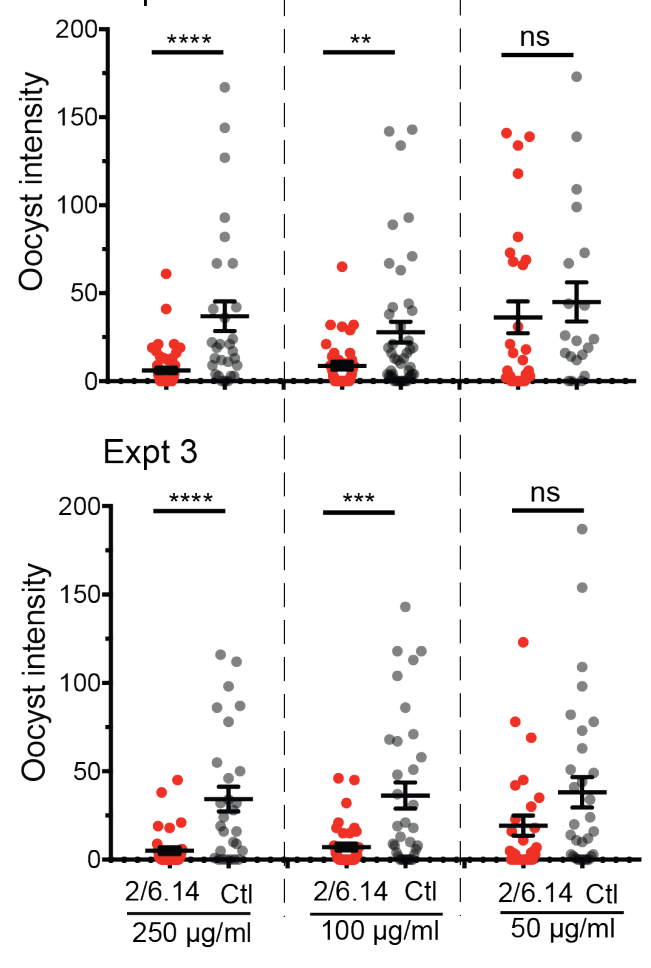

D

\begin{tabular}{lllllll}
\hline & \multicolumn{2}{c}{$250 \mu \mathrm{g} / \mathrm{ml}$} & \multicolumn{2}{c}{$100 \mu \mathrm{g} / \mathrm{ml}$} & \multicolumn{2}{c}{$50 \mu \mathrm{g} / \mathrm{ml}$} \\
\cline { 2 - 7 } & $2 / 6.14$ & $\mathrm{ctl} \mathrm{lgG}$ & $2 / 6.14$ & $\mathrm{ctl} \mathrm{lgG}$ & $2 / 6.14$ & $\mathrm{ctl} \mathrm{lgG}$ \\
\hline Mean intensity & $4.5 \pm 0.9$ & $29.5 \pm 4.0$ & $6.6 \pm 1.1$ & $29.5 \pm 3.6$ & $20.2 \pm 3.7$ & $36.4 \pm 5.0$ \\
Mean prevalence & 38.7 & 84.3 & 49.6 & 82.2 & 69.3 & 80.0 \\
Inhibition in intensity (\%) & $84.6(<0.0001)$ & $77.6(<0.0001)$ & $44.5(0.0025)$ \\
Inhibition in prevalence (\%) & $54.2(<0.0001)$ & $39.7(<0.0001)$ & $13.4(\mathrm{NS})$ & \\
\hline
\end{tabular}

Fig. 3. Immunofluorescent staining and inhibition of $\boldsymbol{P}$. berghei fertilizaton by mAbs. (A) Immunofluorescent staining of microgametes from $P$. berghei. Fixed microgametes were stained with mouse D3 mAbs or control IgG, rabbit anti- $\alpha$-tubulin, secondary Alexa Fluor-488 anti-mouse IgG and Alexa Fluor-594 antirabbit IgG, DAPI, and imaged with epifluorescence. Scale bars $=5 \mu \mathrm{m}$. (B) Fertilization in vitro measured as macrogamete conversion to ookinetes ( $\%$ ookinetes/(ookinetes + macrogametes $))$ in the 
presence of indicated antibodies. Results are mean \pm SEM of 3 independent experiments, analyzed by paired t test: $* * * \mathrm{p}<0.001, * * \mathrm{p}<0.01, \mathrm{~ns}$, non-significant $\mathrm{p}>0.05$. Total number of macrogametes

+ookinetes in all 3 experiments are from right to left at $500 \mu \mathrm{g} / \mathrm{ml}: 139,177,147,104,199$ and 129, and at $250 \mu \mathrm{g} / \mathrm{ml}: 222,120,270,410,275$ and 306. (C and D) Mosquitoes were allowed to feed on antibody or control IgG diluted in infected mouse blood placed in membrane feeders; results are shown from three independent experiments. (C) Oocyst intensities (oocysts per mosquito) are shown as filled circles with means as horizontal bars \pm SEM. $* * * * p<0.0001, * * * p=0.0001-0.001, * * p=0.001-0.01, * p=0.01-0.05$, ns p $>0.05$ by Mann-Whitney test. (D) Summary of intensity and prevalence (\% of infected mosquitoes) with SEM from all 3 experiments. Prevalence and $\mathrm{N}$ values are shown in Fig. S3. Inhibition (\%) was calculated relative to negative control $\mathrm{IgG}$ at the same concentrations. The significance of inhibition is shown in parentheses for intensity (Mann-Whitney test) and prevalence (Fisher's exact test). 
A
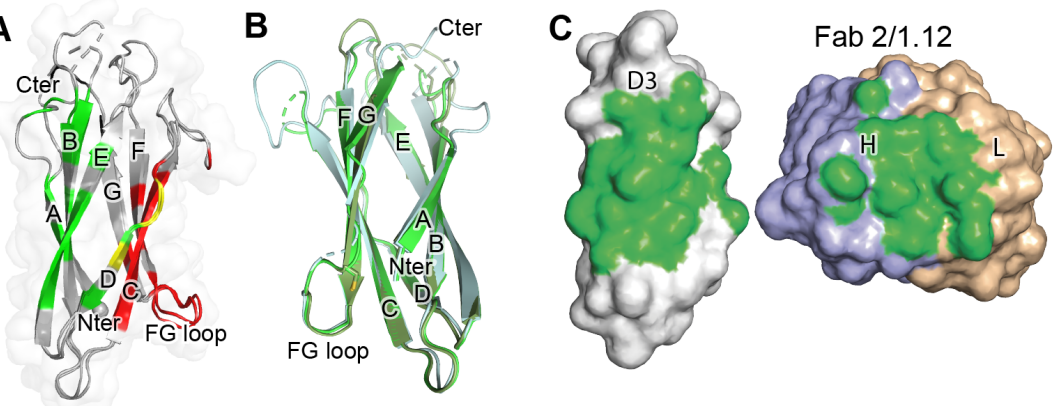
$\mathbf{E}_{\text {RMSD }}$
$\begin{array}{ll}\text { P.ber } & 502 \\ \text { P.kno } & 492 \\ \text { P.viv } & 492 \\ \text { P.mal } & 498 \\ \text { P.ova } & 493 \\ \text { P.fal } & 504\end{array}$

$\mathrm{A} \rightarrow$

$\underset{-310000000000}{\mathrm{~B}} \longrightarrow$

$00000001000000000011---10-1010$

ATITHVTIPNDCASNNSNSNECVLIIHVWNNSKFVGSNFSCSIACTNKETDQLASHINP 560

......T.K..S..Q.E.KD....V.T...TN.......HVI..D.DSGN..T.LG. 550

_...I.T.KN.S.Q.E.KD...V.T...AN......HVI..D...GKI.T..G. 550

_....I.T.K..S.SQT..KD.A..V......NNIS....QVI..D.D.GKI..... 556

_...I.T.E.S.DVQ....I....................SGKI...S. 551

G...I..K..S.OT..K..I.VV.T..N.TI.A...HVL.VD.S.Q.V.T..S. 562

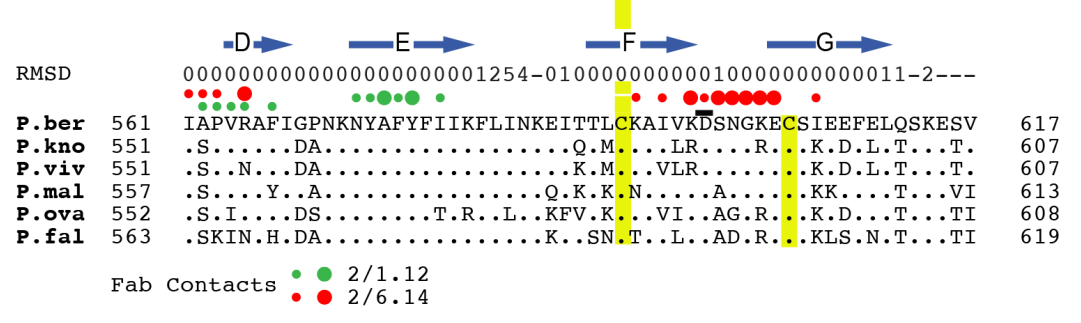

$\mathbf{F}$

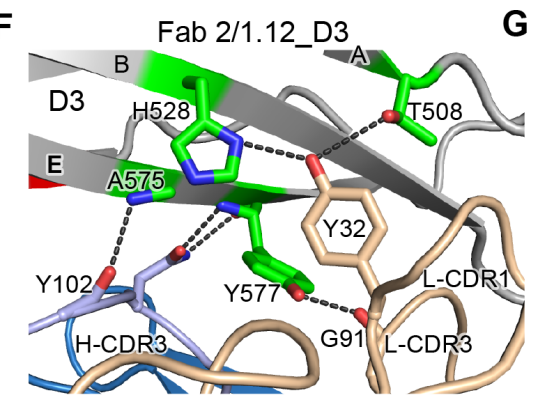

G

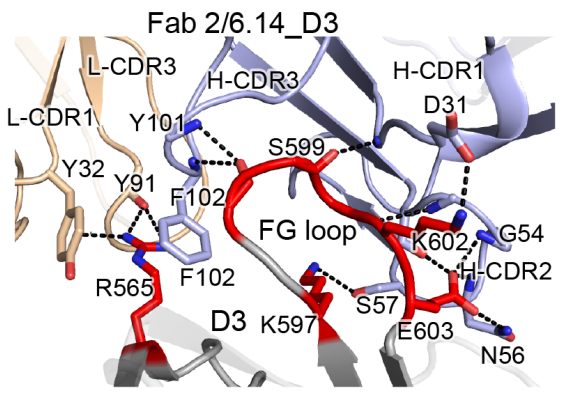

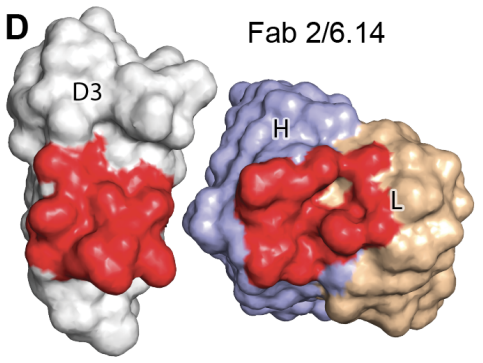

H
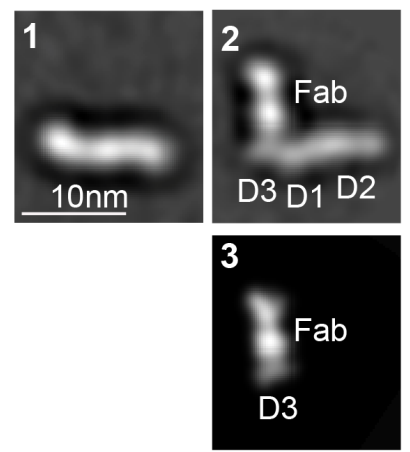

4

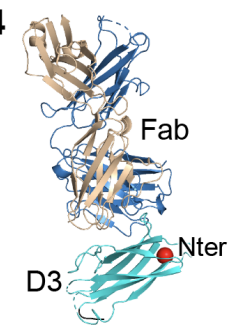

Fig. 4. Structures of HAP2 D3 in complex with 2/6.14 Fab or 2/1.12 Fab. (A) Cartoon representation of D3 with residues colored that contact Fab 2/1.12 (green), Fab 2/6.14 (red) or both Fabs (yellow). (B) Cartoon representations of three independent molecules in the two crystals, excluding the one molecule with multiple conformations. Each molecule is colored in a different variation of green. Dashes symbolize loops with missing density. (C and D) Solvent accessible surfaces of complexes are shown in open book views in which the Fab and D3 components are rotated apart like two facing pages in an open book. Atoms within $5 \AA$ of one another in the two components are colored green (Fab 2/1.12 complex) or red (Fab 2/6.14 complex) and are otherwise white (D3), light blue (Fab H chain), or wheat (Fab L chain). (E) Structural and sequence conservation of D3 and its $2 / 1.12$ and 2/6.14 epitopes. Top line: $\beta$-strands shown as arrows. Second line: Root mean square deviation (RMSD) of C $\alpha$ atom position ( $\AA$ ) among the two independent Fab 2/1.12 - D3 complexes and the Fab 2/6.14-D3 complex with a single conformation, calculated after structure alignment by RaptorX(41). A dash shows positions where residues were defined in only 0 or 1 of the three 3 structures. Third line: Filled circles show Fab contacts (green for 2/1.12 Fab and red for Fab 2/6.14); residues that mediate major interactions ( $>10 \AA^{2}$ of buried solvent accessible surface area or H-bonds) or minor interactions $\left(<10 \AA^{2}\right.$ buried solvent accessible surface) are shown with large or small circles, respectively. Remaining lines show D3 sequence in P. berghei, $P$. knowlesi, $P$. vivax, $P$. malariae, $P$. ovale and $P$. falciparum with identities to $P$. berghei shown as dots. $P$. berghei 
residues in red are wild-type and were mutated to remove N-linked sites and residues in italics were not visualized in any of the three structures. $P$. berghei residues with sidechains mentioned in the text that stabilize hydrogen bonds in the B-C and F-G loops are overlined. Cysteines are highlighted in yellow and connected when disulfide-linked (F and $\mathbf{G})$. Details of D3 interactions with the 2/1.12 Fab (F) and 2/6.14 Fab (G). D3 is silver, Fab H and L chains are light blue and wheat, respectively, and residues with major interactions with Fabs as defined in (E) have carbons colored green (F) or red (G). Dashes show hydrogen bond and pi-cation interactions. (H) The most populated negative stain EM class averages of the HAP2 ectodomain (residues 43-617) alone (panel 1, 1128 particles) and in complex with the 2/6.14 Fab (panel 2, 379 particles). Panel 3 shows the best correlating projection directly from the complex crystal structure. Panel 4 shows a ribbon diagram of the crystal structure in the same orientation. D3 colored cyan, Fab H and L chains colored light blue and wheat, respectively, and N-terminal of D3 shows as a red sphere, $\mathrm{C}$ terminal of D3 colored as black. 
A

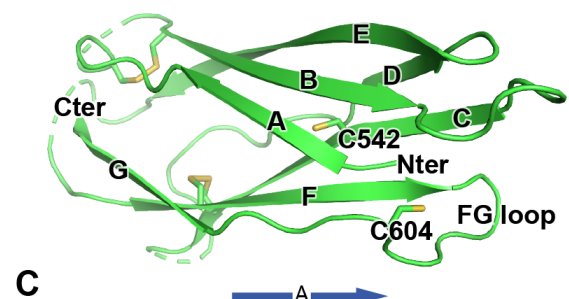

B

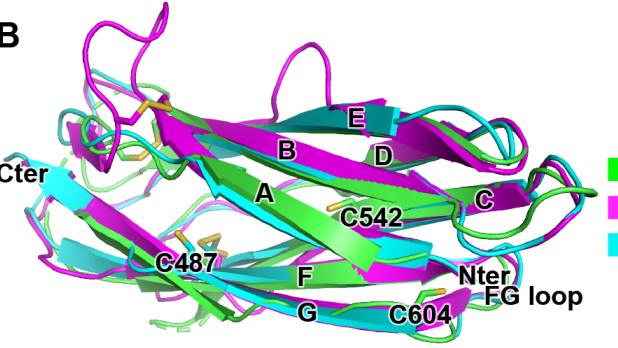

P. berghei

C. reinhardtii

A. thaliana

Russ

$-00 \overline{1} \vec{A}$ $0 \overrightarrow{110000000011-10000000001-12-}$

P.ber 502----ATITHVTI $\dot{P}$ $-1132---0011100000000$

GSNFCSIA-CTNKETTDQLA 555

A. tha 389 QRSPGKIINIAIP--------TFEA----LTQFGVAAVIIKNTG-EVEASYSLTFD-CS-------K 433

C. rei $465-$-SPGKITGAAVCRFAGTSCGGFEA----VAARGYIYVNITNTG-RLDSDYTLTVSNCS-------S 516

RMSD $\quad-1110000011000121 \overrightarrow{1111--110011113012-211100000011110000012110011---~}$

P.ber 556 SHINPIAPVRAFIGPNKNYAF-YFIIKFLINKEI-TTLCKAIVKDSNGKECSIEEFELQS---ー- 613

A. tha 435-GVAFVEEQFFIIKPKAVTTR--SFKLYPTKDQAA-KYICTAILKDSQF SEVDRAECQF STTA--- 493

C. rei 518_NVRPIEARTLAVRAGSAASLDPPMELYVEDQAAAAARTCTVSLYDSVGAVTDSLTLSFYTNATQL 582

Fab $2 / 1.12$ Trimer Arabidopsis thaliana

contacts - 2/6.14 Contacts - Chlamydomonas reinhardtii
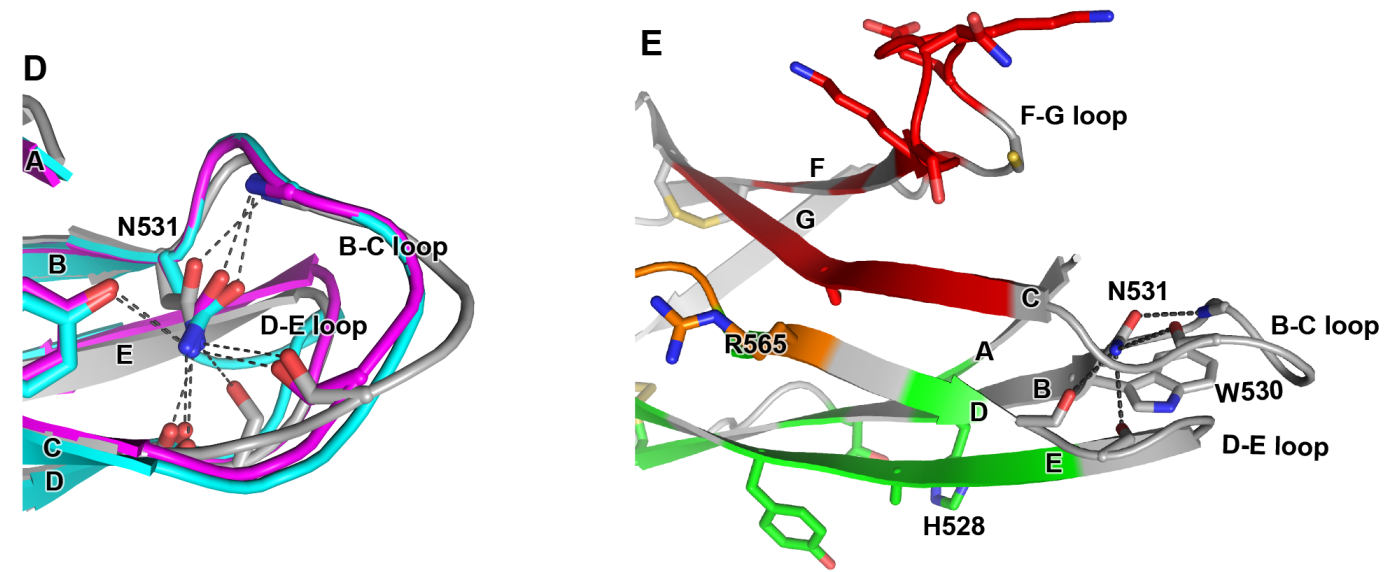

Fig. 5. Structural conservation of domain 3 of HAP2 across phyla. (A) P. berghei HAP2 D3 from the Fab 2/1.12 complex showing the disulfide-bonded and free cysteines. (B) Superimposition of HAP2 D3 from the $P$. berghei 2/6.14 Fab complex and trimeric fusion states of Chlamydomonas reinhardtii (pdb ID:6DBS) and Arabidopsis thaliana (pdb ID: 5ow3). Structures were aligned using RaptorX (41). (C) Structure-based sequence alignment from the superimposition shown in (A). $\beta$-strands and root mean square deviation (RMSD) $(\AA)$ of $\mathrm{C} \alpha$ atom positions are shown above the sequences. Green and red filled circles above the $P$. berghei sequence show Fab 2/1.12 and Fab 2/6.14 contacts, respectively as defined in Fig. 4E legend. In the $P$. berghei sequence, the three residues in red mark residues that were mutated to remove N-linked sites. Orange and blue filled large and small circles above Arabidopsis thaliana and Chlamydomonas reinhardtii sequences mark residues buried in trimer contacts with $>10 \AA^{2}$ burial or a hydrogen bond or $<10 \AA^{2}$, respectively. Solvent accessible surface area burial was calculated with PISA (42). (D) Asn-531, present in an N-glycosylation sequon in the P. berghei sequence, forms stabilizing hydrogen bonds to the backbones of the $\mathrm{B}-\mathrm{C}$ and $\mathrm{D}-\mathrm{E}$ loops, a function that is conserved in HAP2 in other phyla. The color code is the same as in panel A, except $P$. berghei is in silver. (E) Asn-531 locates near $\mathrm{mAb}$ epitopes. Asn-531 and sidechains or backbones to which it hydrogen bonds are shown in stick. Residues with major or minor contacts with Fabs as defined in Fig. 4E legend are shown with both 
bioRxiv preprint doi: https://doi.org/10.1101/2021.10.22.465389; this version posted October 24, 2021. The copyright holder for this preprint (which was not certified by peer review) is the author/funder, who has granted bioRxiv a license to display the preprint in perpetuity. It is made available under aCC-BY 4.0 International license.

backbone and sidechain, or backbone only, respectively, and colored according to the Fig. 4E legend and the key. Residues in both epitopes are in orange. 

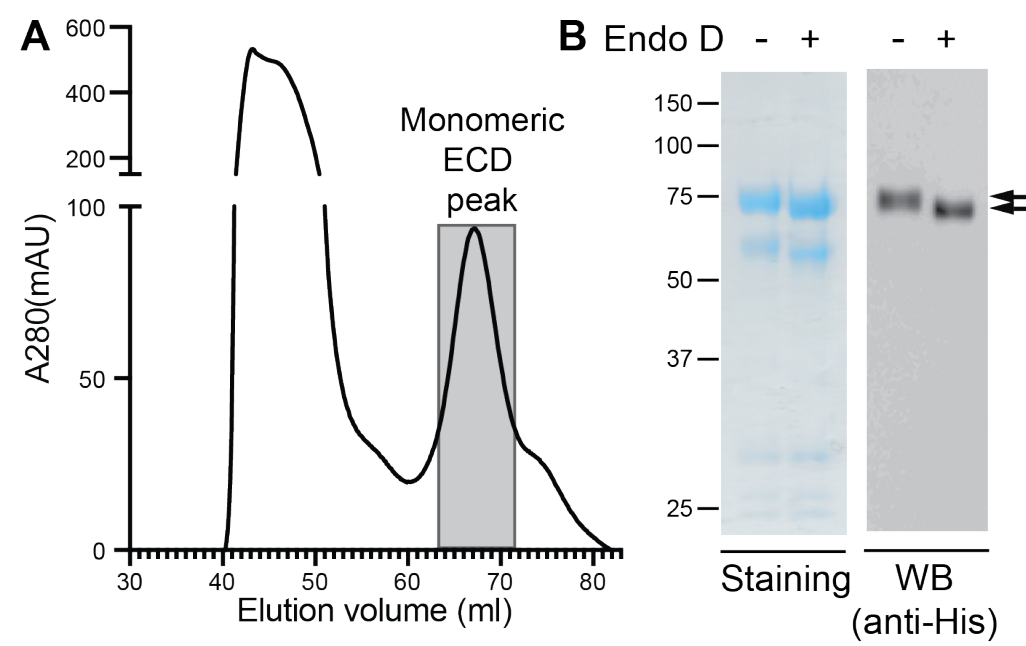

E Affinity and kinetics comparisons.

\begin{tabular}{llrcr}
\hline Analyte & Immobile & $\mathbf{K}_{\mathbf{D}}(\mathbf{n M})$ & $\mathbf{k}_{\text {on }}\left(\mathbf{1 0}^{4} \mathbf{M}^{-1} \mathbf{s}^{-1}\right)$ & $\mathbf{k}_{\text {off }}\left(\mathbf{1 0}^{-4} \mathbf{s}^{-1}\right)$ \\
\hline D3 & $2 / 1.12$ & $5.2 \pm 0.5$ & $17.5 \pm 0.9$ & $9.0 \pm 0.4$ \\
2/1.12 & D3 & $5.9 \pm 0.2$ & $8.5 \pm 0.2$ & $5.0 \pm 0.1$ \\
ECD & $2 / 1.12$ & $106 \pm 2.0$ & $0.77 \pm 0.01$ & $8.1 \pm 0.1$ \\
2/1.12 & ECD & $11.7 \pm 8.0$ & $9.6 \pm 6.7$ & $5.9 \pm 0.1$ \\
& & & & \\
D3 & $2 / 6.14$ & $6.0 \pm 2.3$ & $114 \pm 41$ & $59 \pm 1.1$ \\
2/6.14 & D3 & $10.2 \pm 0.2$ & $30.3 \pm 1.0$ & $32.2 \pm 1.2$ \\
ECD & $2 / 6.14$ & $22.9 \pm 0.9$ & $11.9 \pm 0.5$ & $27.2 \pm 0.2$ \\
2/6.14 & ECD & $50.5 \pm 4.2$ & $7.2 \pm 0.8$ & $36.1 \pm 1.0$ \\
\hline
\end{tabular}
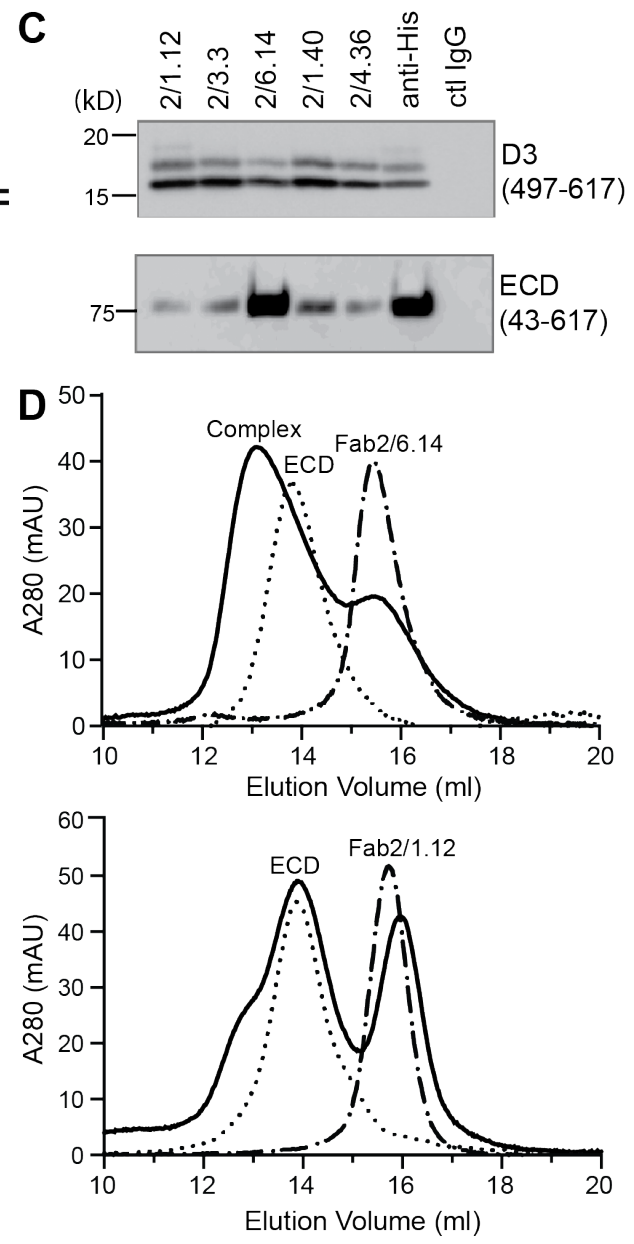

Fig. 6. Antibody binding to the HAP2 ectodomain. (A) Gel filtration profile of Ni-NTA affinity-purified ectodomain (residues 43-617) on a Hiload 16/600 Superdex 200 column. (B) Reducing SDS 7.5\% PAGE and Coomassie blue staining and Western blot (WB) of the peak eluted at $67.2 \mathrm{~mL}$ (shaded in A). Aliquots were treated with or without Endo D to remove N-linked glycans. Arrows indicate ectodomain bands before and after Endo D treatment. The HAP2 ectodomain contains 11 putative N-glycosylation sites; however, Endo D treatment reduced the mass by only $\sim 3 \mathrm{kD}$. It is unknown how many sites are Nglycosylated and whether all sites are accessible to Endo D. (C) Immunoprecipitation. Reactivity of D3 mAbs to HAP2 ectodomain (bottom) in comparison to D3 (top). $2 \mu \mathrm{g}$ purified ectodomain from (A), without Endo D treatment, was subjected to immunoprecipitation with the indicated D3 mAbs, anti-His mAb 1/5.13 or control IgG. D3 (497-617, containing 3 putative glycosylation sites) was immunoprecipitated from culture supernatants of S2 transfectants. Multiple D3 bands are different glycoforms. (D) Analysis of formation of PbHAP2 ectodomain complex with 2/6.14 Fab (top) and 2/1.12 $\mathrm{Fab}$ (bottom) by gel filtration. Ectodomain and $\mathrm{Fab} 2 / 6.14$ or Fab 2/1.12 were mixed in $20 \mathrm{mM}$ Tris-HCl $\mathrm{pH} 8.0,500 \mathrm{mM} \mathrm{NaCl}$ at 1:1 molar ratios and incubated at $4^{\circ} \mathrm{C}$ for 1 hour. Mixtures was then subjected to gel filtration on a Superdex 200 10/300 GL column in the same buffer. Elution profiles of the mixture, ectodomain or Fabs are shown as solid, dotted, or dashed lines, respectively. (E) $K_{D}$ values $\left(k_{\text {off }} / k_{\text {on }}\right)$ and kinetic values measured by surface plasmon resonance. Values are mean \pm difference of the means from 2 independent experiments. D3 contained residues 502-617 with N516T, S533N and N539Q mutations and the ectodomain (ECD) contained residues 43-617 without Endo D treatment. 
A

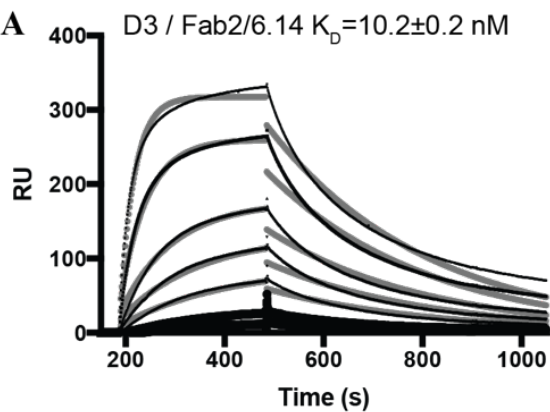

C
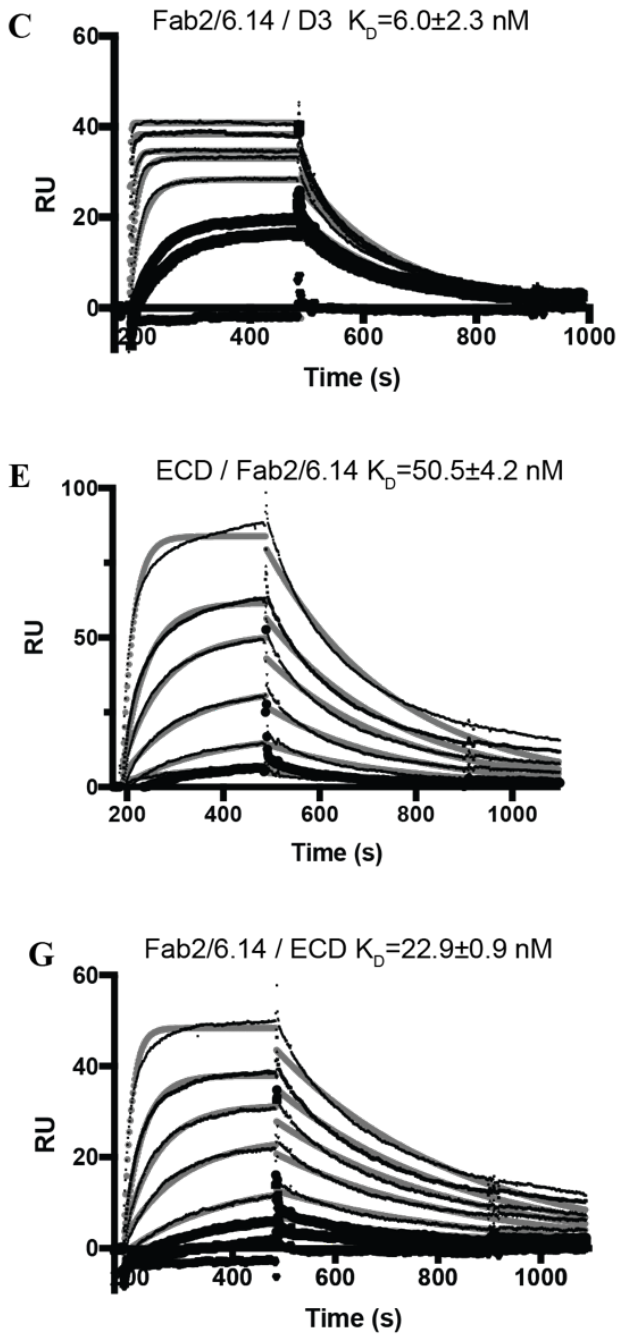
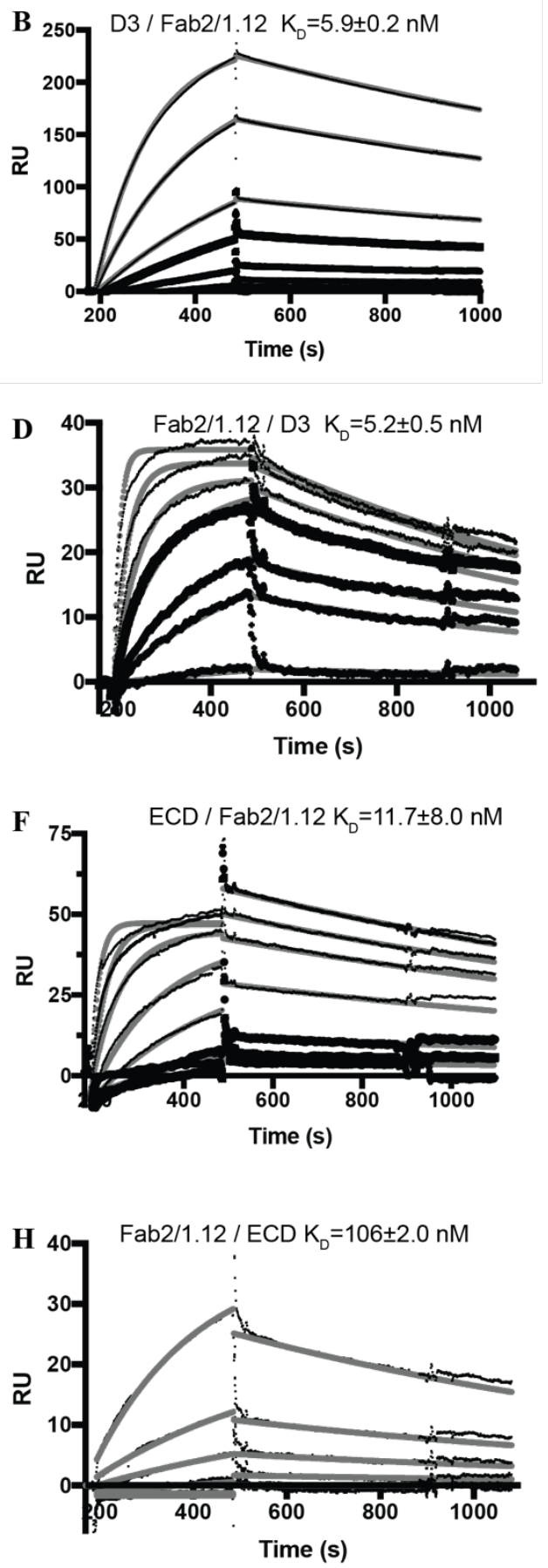

Figure S1. (Related to Figs 2 and 6). Surface plasmon resonance (SPR) analysis of binding

Interactions of Fab 2/6.14 and Fab 2/1.12 with PbHAP2 D3 and monomeric ectodomain. (A-

F) SPR sensorgrams are shown in thin black lines and fits in thick gray lines. Concentrations used for Fab 2/6.14 (A) and Fab2/1.12 (B) were 100, 50, 20, 10, 5, 2, $1 \mathrm{nM}$. Concentrations used for 
D3 (C) were 1000, 500, 200, 100, 50, 20, 10, $5 \mathrm{nM}$ and for D3 (D) were 500, 200, 100, 50, 20, 5

nM. Concentrations used for Fab 2/6.14 (E) and Fab2/1.12 (F) were 500, 200, 100, 50, 20, 10, 5

nM. Concentrations used for ectodomain $(\mathrm{G}$ and $\mathrm{H})$ were 300, 120, 60, 30, 12, 6, $3 \mathrm{nM}$. 


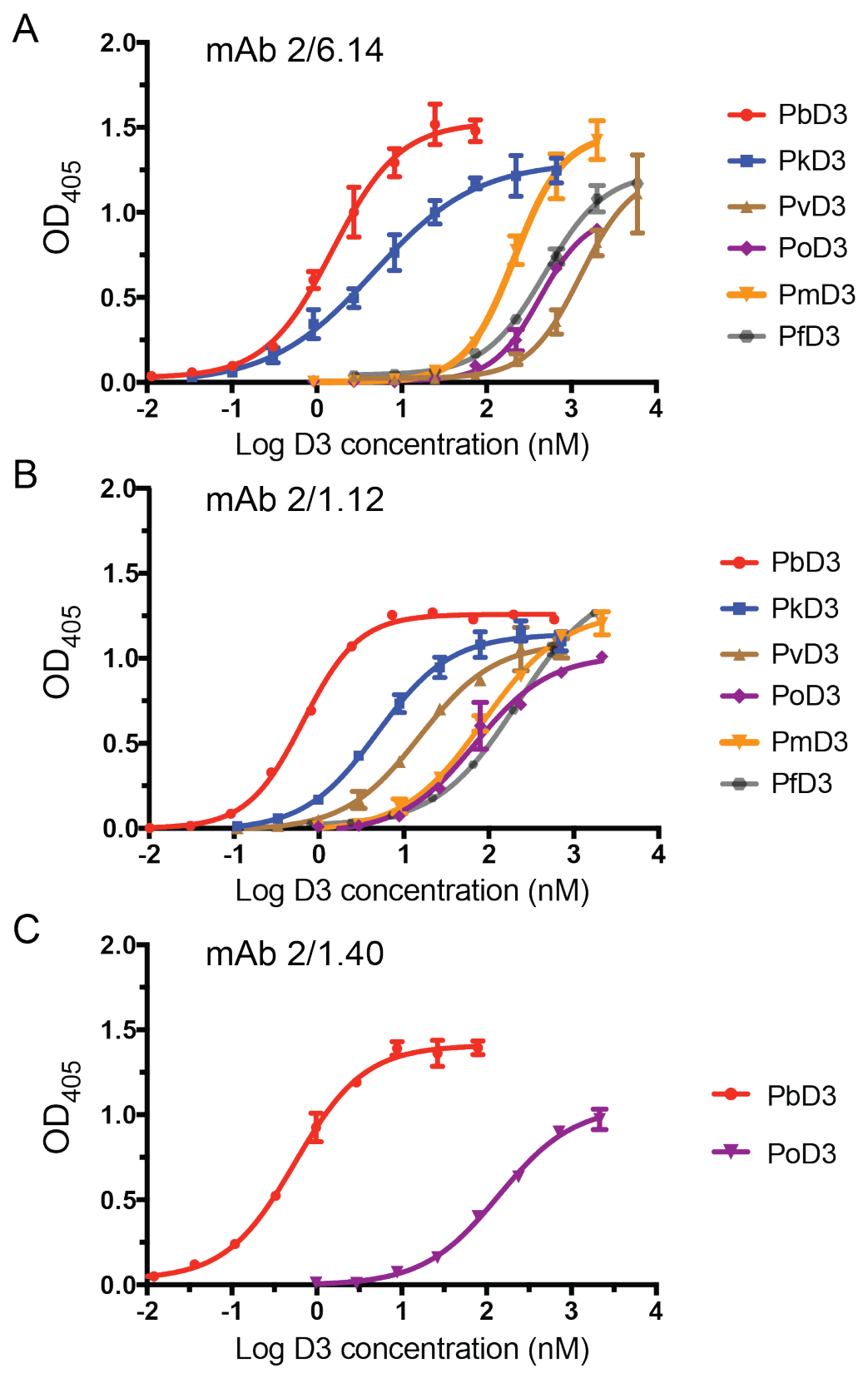

Figure S2. (Related to Figure 2). Titration of binding of HAP2 D3 from Plasmodium species to

immobilized mAbs. Elisa plates on which mAb 2/6.14 (A), 2/1.12 (B) or 2/1.40 (C) at $5 \mathrm{ug} / \mathrm{ml}$ were immobilized were then incubated with purified, His-tagged D3 from Plasmodium spp. at varying concentrations. Binding was detected by incubation with HRP-conjugated anti-His. Nonlinear titration curve fitting was performed using GraphPad Prism 7 software. Data shown are mean $\pm \mathrm{SD}$ of triplicate measurements in one representative experiment. 

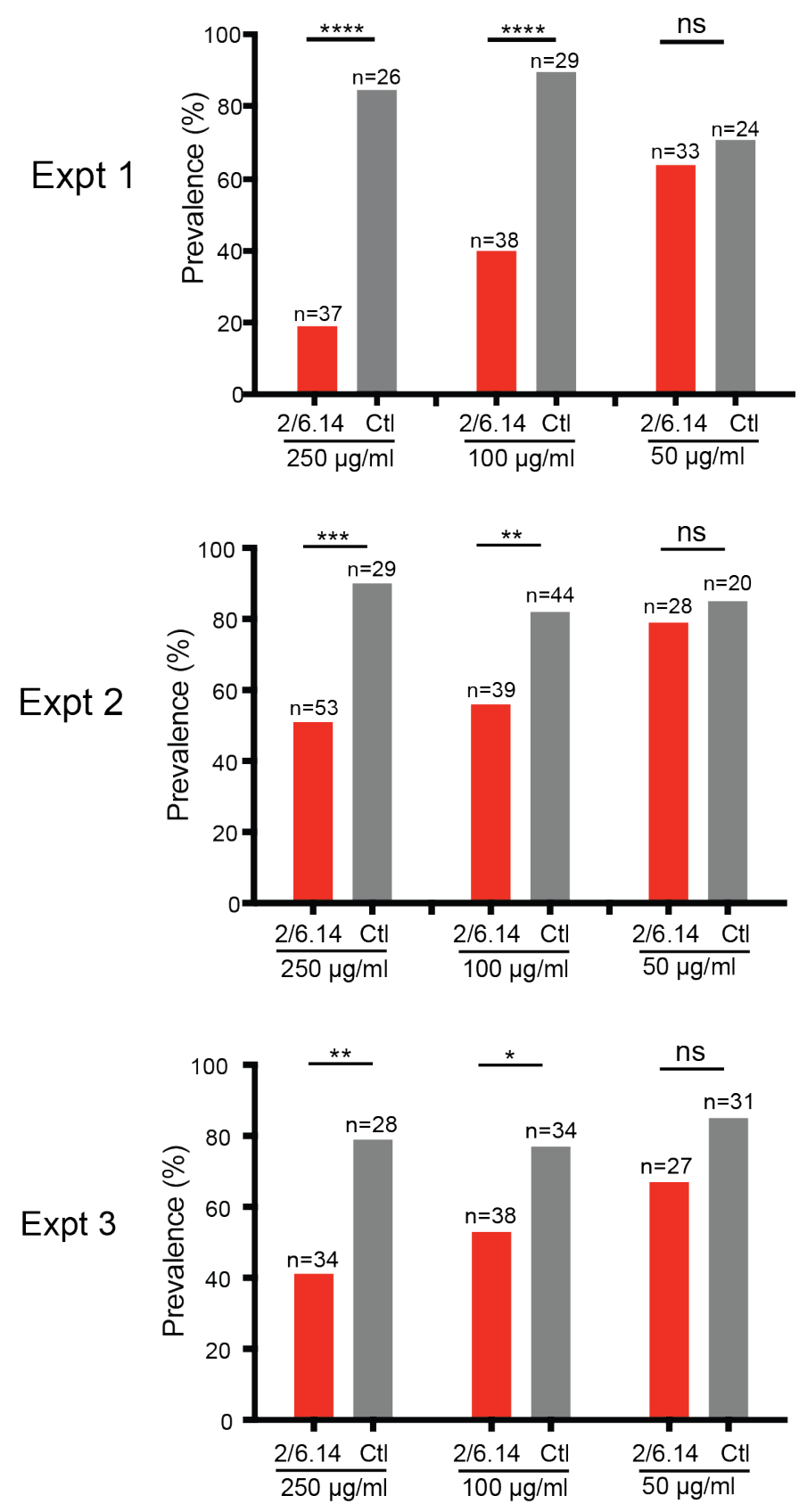

Figure S3. (Related to Figure 3C and 3D). Transmission blocking activity of mAb 2/6.14 in

standard membrane feeding assay. Data shown are prevalence ( $\%$ of infected mosquitoes) in 3

independent experiments. Number of mosquitoes per group is shown above bars. Significance

was determined by Fisher's exact test. $* * * * p<0.0001, * * * \mathrm{p}=0.0001-0.001,{ }^{* *} \mathrm{p}=0.001-0.01$, ${ }^{*} \mathrm{p}=0.01-0.05, \mathrm{~ns} \mathrm{p}>0.05$. 

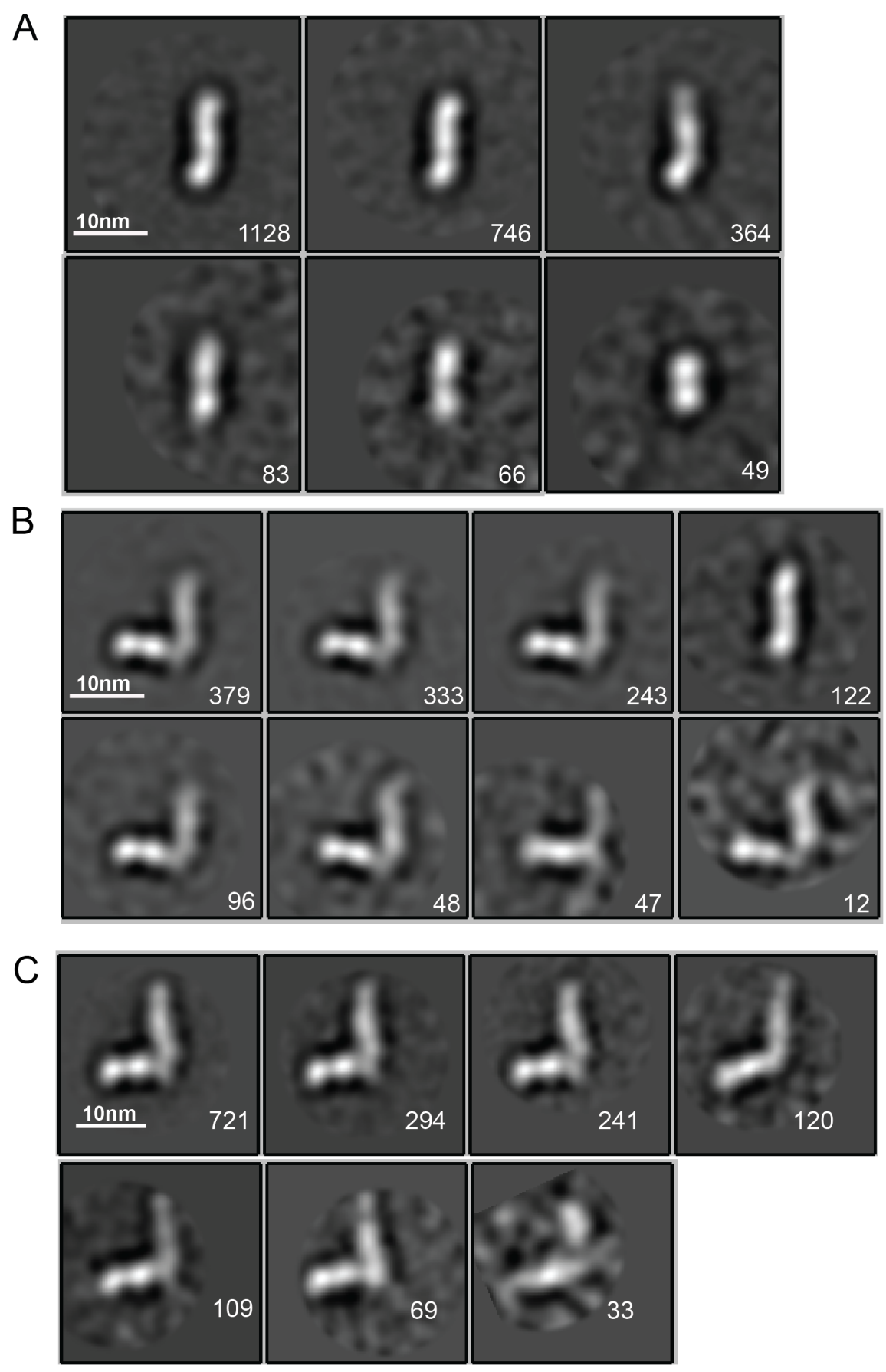

Figure S4. (Related to Figure 6). EM class averages. Classes are from RELION classification of negatively stained particles of the Hap2 ectodomain, residues 43-617 (A) or Fab 2/6.14 complexes with the Hap2 ectodomain, residues 43-617 (B) or with the Hap2 ectodomain, residues 61-611 (C). Particle numbers are shown for each class average. Scale bars (10 nm) are shown as a white line. 

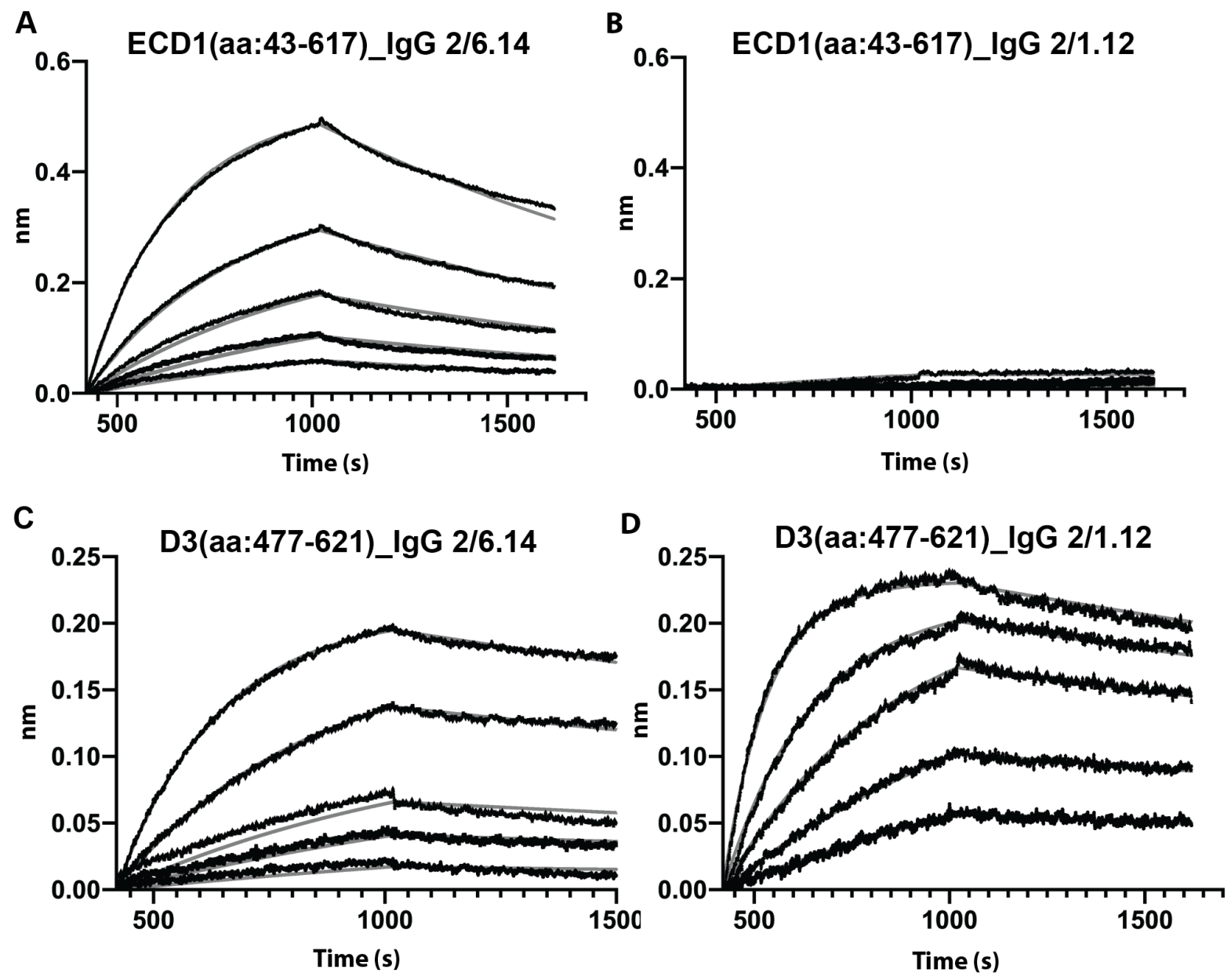

E Affinity and kinetics comparisons.

\begin{tabular}{lllcr}
\hline Analyte & $\mathbf{I m m o b i l e}$ & $\mathbf{K}_{\mathbf{D}}(\mathbf{n M})$ & $\mathbf{k}_{\text {on }}\left(\mathbf{1} \mathbf{0}^{4} \mathbf{M}^{-1} \mathbf{s}^{-1}\right)$ & $\mathbf{k}_{\text {off }}\left(\mathbf{1 0}^{-4} \mathbf{s}^{-1}\right)$ \\
\hline D3 & $\operatorname{lgG} 2 / 6.14$ & $0.64 \pm 0.04$ & $43.1 \pm 0.1$ & $2.77 \pm 0.01$ \\
ECD1 & $\operatorname{lgG} 2 / 6.14$ & $18.4 \pm 0.1$ & $3.94 \pm 0.01$ & $7.24 \pm 0.01$ \\
D3 & $\operatorname{lgG} 2 / 1.12$ & $1.68 \pm 0.01$ & $13.3 \pm 0.2$ & $2.23 \pm 0.01$ \\
ECD1 & $\operatorname{lgG} 2 / 1.12$ & No binding & & \\
\hline
\end{tabular}

Figure. S5. Biolayer interferometry (BLI) analysis of binding Interactions of PbHAP2 D3 and monomeric ectodomain with IgG 2/6.14 and IgG 2/1.12. Concentrations used for (A-B) monomeric ectodomain (ECD1) were 100, 50, 25, 12.5, 6.25, $0 \mathrm{nM}$.

Concentrations used for PbeD3 (C-D) were 16, 8, 4, 2, 1, 0 nM. Response curves of BLI are in black and fitting curves in gray. (E) $\mathrm{K}_{\mathrm{D}}$ values $\left(\mathrm{k}_{\mathrm{off}} / \mathrm{k}_{\mathrm{on}}\right)$ and kinetic values measured by BLI. The kinetic parameters were fit globally to sensorgrams at different 
analyte concentrations. Errors are SE(standard error) from nonlinear least square fits. All experiments were repeated at least twice with essentially identical results. PbD3

contained the residues 477-621, and the ECD1 contained residues 43-617, both of the PbD3 and ECD1 are without Endo D treatment. 

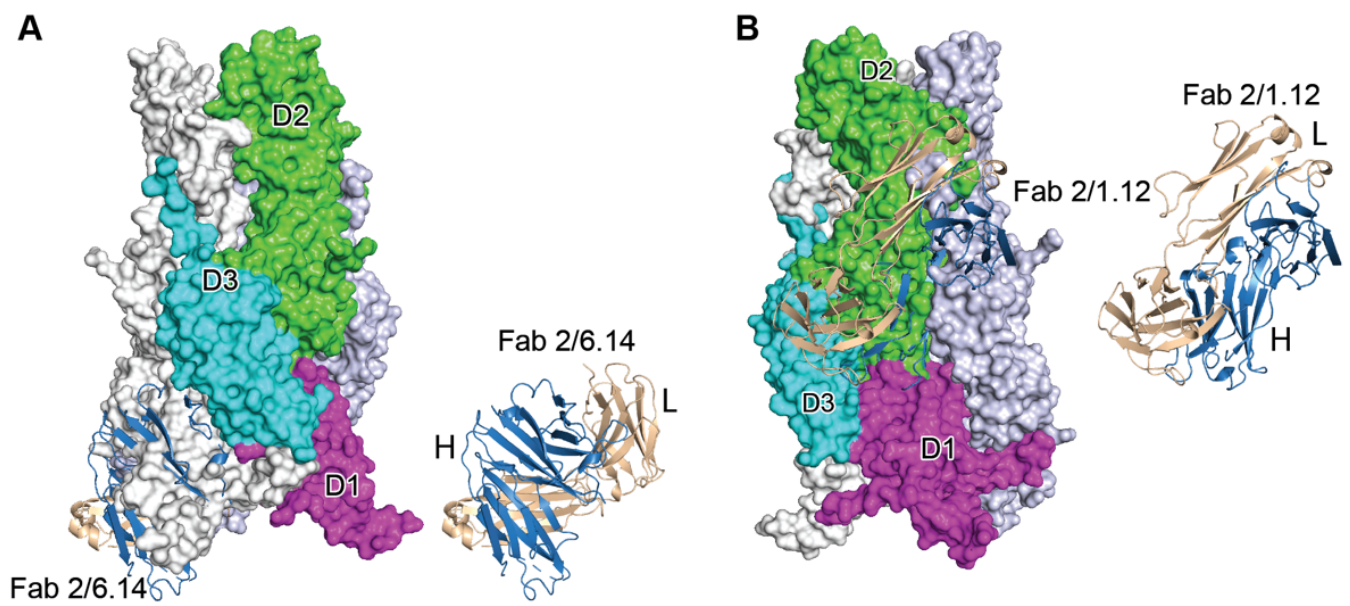

Figure. S6. Steric clashes of mAbs 2/6.14 and 2/1.12 with the postfusion HAP2

conformation. Complexes of PbHAP2 D3 with the 2/6.14 Fab (A) and 2/1.12 Fab (B) were superimposed on D3 of the postfusion C. reinhardtii HAP2 trimer. The postfusion HAP2 structure is shown as a surface and colored by domain in one monomer; other monomers are white and lightblue (pdb ID:6DBS). Fab H and L chains are light blue and wheat, respectively, and are shown alone to the right to emphasize how much of each Fab is buried. 

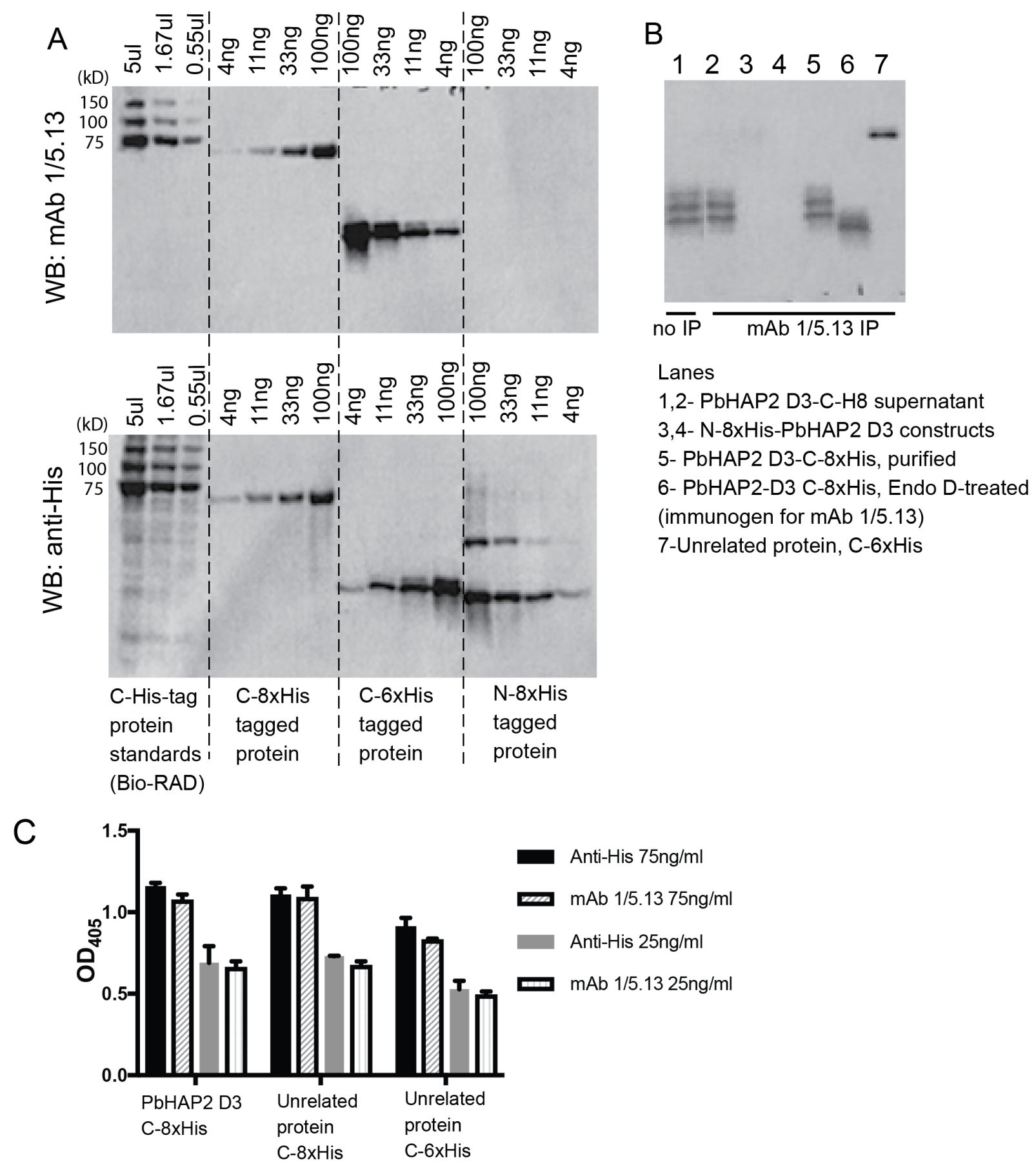

Figure S7. Reactivity of mAb 1/5.13 to fusion proteins with His tags. (A) Western blot. C-, N-terminal His tagged purified proteins (C-8xHis Chlamydomonas HAP2 ectodomain, C-6xHis Plasmodium falciparum TRAP fragment, N-8xHis-pro-TGFß1) or Bio-RAD Precision Plus All Blue Protein Standards (Cat \#1610373, the 75, 100 and 150kD proteins are C-His tagged) were 
loaded at the indicated concentration per lane and run on $10 \%$ reducing SDS-PAGE. The blot was detected with $1 \mu \mathrm{g} / \mathrm{ml} \mathrm{mAb} 1 / 5.13$ or THETM His Tag Antibody (Genscript, Cat. No. A00186). THE ${ }^{\mathrm{TM}}$ His Tag Antibody detected C- and N-His-tagged proteins with correct molecular sizes, whereas mAb 1/5.13 didn't detect the N-His protein at the concentrations tested. (B) Immunoprecipitation. $3 \mu \mathrm{g}$ of purified proteins (lanes 3 -7) or $100 \mu 1$ of cultural supernatant of transfectants (lane 2) were subjected to immunoprecipitation with mAb 1/5.13 (8 ug). Immunoprecipitates were analyzed by Western blot using rabbit anti-His followed by HRP-antirabbit. (C) Elisa. Plates were coated with $5 \mu \mathrm{g} / \mathrm{ml} \mathrm{C-His} \mathrm{tagged} \mathrm{PbHAP} 2 \mathrm{D} 3$ or proteins unrelated to $\mathrm{PbHAP} 2 \mathrm{D} 3$, incubated with $\mathrm{mAb} 1 / 5.13$ or $\mathrm{THE}^{\mathrm{TM}}$ His Tag Antibody at the indicated concentrations. 
Table S1. Statistics of X-ray diffraction and structure refinement of PbHAP2 domain 3 (D3) complexed with 2/6.14 Fab or 2/1.12 Fab

\begin{tabular}{|c|c|c|}
\hline & D3-2/6.14 Fab & D3-2/1.12 Fab \\
\hline \multicolumn{3}{|l|}{ Data collection statistics } \\
\hline Space group & P222 & $\mathrm{P} 2_{1}$ \\
\hline$\alpha, \beta, \gamma,{ }^{\circ}$ & $90,90,90$ & $90,95.8,90$ \\
\hline Unit cell $(\mathrm{a}, \mathrm{b}, \mathrm{c}), \AA$ & $78.3,122.6,168.5$ & $43.9,187.1,74.4$ \\
\hline Resolution range $(\AA)$ & $50.0-2.80(2.87-2.80)^{\mathrm{a}}$ & $50.0-2.10(2.15-2.10)$ \\
\hline Completeness (\%) & $99.3(99.5)$ & $98.4(96.9)$ \\
\hline Number unique reflections & $40,741(2,938)$ & $68138(4,935)$ \\
\hline Redundancy & $3.8(4.0)$ & $3.6(3.7)$ \\
\hline $\mathrm{R}_{\text {merge }}(\%)^{\mathrm{b}}$ & $5.7(70.2)$ & $8.3(153)$ \\
\hline $\mathrm{I} / \sigma(\mathrm{I})$ & $12.8(2.2)$ & $8.42(0.52)$ \\
\hline $\mathrm{CC}_{1 / 2}(\%)^{\mathrm{c}}$ & $99.8(90.9)$ & $99.7(10.9)$ \\
\hline Wavelength $(\AA)$ & 1.0332 & 1.0332 \\
\hline \multicolumn{3}{|l|}{ Refinement statistics } \\
\hline $\mathrm{R}_{\text {work }}(\%)^{\mathrm{d}}$ & $25.3(33.7)$ & $18.97(37.3)$ \\
\hline $\mathrm{R}_{\text {free }}(\%)$ & $29.2(34.8)$ & $23.27(40.5)$ \\
\hline Bond RMSD $(\AA)$ & 0.003 & 0.003 \\
\hline Angle RMSD $\left(^{\circ}\right)$ & 0.548 & 0.603 \\
\hline $\begin{array}{l}\text { Ramachandran plot }{ }^{\mathrm{e}} \\
\text { (Favored/allowed/outlier) }\end{array}$ & $91.5 / 7.6 / 0.9$ & $95.93 / 4.07 / 0.1$ \\
\hline \multicolumn{3}{|l|}{ Number of atoms } \\
\hline Protein & 10,310 & 8,183 \\
\hline Ligand & 22 & 54 \\
\hline Water & 18 & 353 \\
\hline \multicolumn{3}{|l|}{ B factor } \\
\hline Protein & 128.6 & 72.7 \\
\hline Ligand & 112.8 & 112.9 \\
\hline Water & 138.0 & 64.9 \\
\hline \multicolumn{3}{|l|}{ Molprobity percentile } \\
\hline (Clash/Geometry) & 98/97 & 99/97 \\
\hline PDB & 7LR3 & 7LR4 \\
\hline
\end{tabular}

${ }^{\mathrm{a}}$ The numbers in parentheses refer to the highest resolution shell.

${ }^{\mathrm{b}} \mathrm{R}_{\text {merge }}=\Sigma \mathrm{h} \Sigma \mathrm{i}|\mathrm{Ii}(\mathrm{h})-<\mathrm{I}(\mathrm{h})>| / \Sigma \mathrm{h} \Sigma \mathrm{i} \mathrm{Ii}(\mathrm{h})$, where $\mathrm{Ii}(\mathrm{h})$ and $<\mathrm{I}(\mathrm{h})>$ are the $\mathrm{i}^{\text {th }}$ and mean measurement of the intensity of reflection $h$.

${ }^{c}$ Pearson's correlation coefficient between average intensities of random half-datasets for each unique reflection ${ }^{38}$.

${ }^{\mathrm{d}} \mathrm{R}_{\text {factor }}=\Sigma \mathrm{h}||$ Fobs (h)|-|Fcalc (h) ||$/ \Sigma \mathrm{h} \mid$ Fobs (h)|, where Fobs (h) and F calc (h) are the observed and calculated structure factors, respectively. No I/ $\sigma(\mathrm{I})$ cutoff was applied.

${ }^{\mathrm{e}}$ Calculated with MolProbity ${ }^{32}$. 